\title{
COMPUTATIONAL APPROACHES IN OPTICS OF FRACTAL CLUSTERS
}

\author{
Vadim A. MARKEL and Vladimir M. SHALAEV \\ Department of Physics, New Mexico State University, \\ Las Cruces, NM 88003, USA \\ Email:vmarkel@nmsu.edu
}

\begin{abstract}
Theoretical and computational approaches to investigating optical properties of fractal clusters built from many small identical particles are reviewed and illustrated by numerical examples.
\end{abstract}

\section{Introduction}

The physical and geometrical properties of fractal clusters attracted a persistently growing attention of researchers in the past two decades. ${ }^{1-3}$ The reason for this is two-fold. First, it turns out that, in many cases, natural processes of aggregation of small particles lead to formation of fractal clusters rather than regular structures. ${ }^{4,5}$ Examples include aggregation of colloidal particles in solutions ${ }^{6-10}$ formation of fractal soot from little carbon spherules in the process of incomplete combustion of carbohydrates, ${ }^{11-16}$ and growth of cold-deposited self-affine films. ${ }^{17,18}$ The second reason is that the properties of fractal clusters are very rich in physics and different from those of either bulk material, or isolated particles (monomers). ${ }^{19}$ In this chapter we focus on theoretical and computational approaches to calculating optical characteristics of fractal clusters, such as the optical cross sections, and review some of the results.

The most simple and extensively used model for fractal clusters is a collection of identical spherically symmetrical particles (monomers) that form a selfsupporting geometrical structure. It is convenient to think of the monomers as of identical rigid spheres that form a bond on contact. A cluster is selfsupporting if each monomer is attached to the rest of the cluster by one or more bonds. Fractal clusters are classified as geometrical (built as a result of a deterministic iteration process) or random. Most clusters in nature are random.

The fractal dimension of a cluster, $D$, can be found from the relation between the number of particles in a cluster, $N$, and its gyration radius, $R_{g}$ :

$$
N=\left(R_{g} / R_{0}\right)^{D},
$$

where $R_{0}$ is a constant of the order of the minimum separation between 
monomers. Another definition of the fractal dimension utilizes the pair densitydensity correlation function $\langle\rho(\mathbf{r}) \rho(\mathbf{r}+\mathbf{R})\rangle$ :

$$
\langle\rho(\mathbf{r}) \rho(\mathbf{r}+\mathbf{R})\rangle \propto R^{D-3} \text { if } R_{0} \ll r \ll R_{g} .
$$

Typically, both definitions give very close values for the fractal dimension. However, a difference can be seen in very large clusters, when multiscaling can be present. ${ }^{20,21}$

The major models for computer simulation of random fractal clusters are the cluster-cluster aggregation (Meakin model) ${ }^{22,23}$ the diffusion limited aggregation (Witten-Sander model), ${ }^{24}$ and the percolation model. ${ }^{25}$ The first two models involve aggregation processes and, therefore, are dynamical. The third model is static in nature. The majority of natural clusters can be accurately described by one of these models with some variations. More details about the aggregation algorithms as well as further references can be found in the reviews by Jullien and Botet. ${ }^{4,5}$

Random fractal clusters are very complex systems built from simple elementary blocks. It is important to emphasize that the rich and complicated properties of fractal clusters are determined by their geometrical structure rather than by the structure of each elementary block (monomer). In the formulation of a typical problem in optics of fractal clusters, the properties of monomers and the law of their interaction with the incident field and with each other are known, while the properties of a cluster as a whole must be found.

The early advances in the study of nonresonant scattering of light and Xrays by fractal clusters were due to Bale and Schmidt, ${ }^{26}$ Berry and Percival ${ }^{27}$ and Martin and Hurd. ${ }^{28}$ Since that, the theory of nonresonant scattering by fractals was developed in great detail. ${ }^{12,13,15,21,29-36}$

The nontrivial fluctuative nature of fractals is fully manifested when one considers the resonant optical properties of fractal clusters. The foundations of the theory of resonant interaction of light with fractals were built by Butenko, Shalaev and Stockman, ${ }^{37,38}$ Markel, Muratov, Stockman and George, ${ }^{39}$ Shalaev, Botet and Jullien ${ }^{40}$ and Stockman, George and Shalaev. ${ }^{41}$ Localization of light and strong enhancement of local fields in fractals were intensively studied $^{37-39,42-51}$ As was originally predicted, ${ }^{38,42,44}$ strong fluctuations of local fields in fractals result in giant enhancement of nonlinear susceptibilities. ${ }^{19,46,52}$ A $10^{6}$ enhancement of the degenerate four-wave mixing in fractal silver clusters was observed experimentally. ${ }^{53,54}$ Wavelength- and polarizationselective spectral holes in the absorption spectra of fractal metal clusters in colloid solutions were induced by intense laser pulses. ${ }^{6,9,55}$ Other strongly enhanced nonlinearities in absorption and refraction by fractal clusters were also 
observed. ${ }^{10}$

Practically, all the existing analytical solutions in optics of random fractal clusters are approximate, and the limits of their applicability are usually not obvious a priori. In many instances, such solutions don't exist at all. This makes numerical methods to be of great importance. While many theoretical methods, such as various scaling theories, ${ }^{39-41,44,47,49,50,56,57}$ explicitly make use of the fractal geometry of clusters, most computational methods are designed for clusters of arbitrary geometry. However, when one is interested in performance characteristics of a numerical method, such as convergence, accuracy or accumulation of the round off errors, the fractal geometry can become important.

\section{Basic Theoretical Approaches}

In this Section we consider a cluster of $N$ monomers located in the points $\mathbf{r}_{i}, i=1, \ldots, N$ and interacting with an incident plane monochromatic wave of the form

$$
\mathbf{E}_{i n c}(\mathbf{r}, t)=\mathbf{E}_{0} \exp (i \omega t-i \mathbf{k} \cdot \mathbf{r}) .
$$

The factor $\exp (i \omega t)$ is common for all time varying fields and will be omitted below.

\subsection{Coupled Dipoles}

In this model, each monomer in a cluster is considered to be a point dipole with polarizability $\alpha$, located at the point $\mathbf{r}_{i}$ (at the center of the respective spherical monomer). The dipole moment of the $i$ th monomer, $\mathbf{d}_{i}$, is proportional to the local field at the point $\mathbf{r}_{i}$ which is a superposition of the incident field and all the secondary fields scattered by other dipoles. Therefore, the dipole moments

of the monomers are coupled to the incident field and to each other as described by the coupled dipole equation $(\mathrm{CDE})$ :

$$
\mathbf{d}_{i}=\alpha\left[\mathbf{E}_{i n c}\left(\mathbf{r}_{i}\right)+\sum_{j \neq i}^{N} \hat{G}\left(\mathbf{r}_{i}-\mathbf{r}_{j}\right) \mathbf{d}_{j}\right] .
$$

Here the term $\hat{G}\left(\mathbf{r}_{i}-\mathbf{r}_{j}\right) \mathbf{d}_{j}$ gives the dipole radiation field created by the dipole $\mathbf{d}_{j}$ at the point $\mathbf{r}_{i}$, and $\hat{G}(\mathbf{r})$ is the regular part of the free space dyadic Green's function:

$$
G_{\alpha \beta}(\mathbf{r})=k^{3}\left[A(k r) \delta_{\alpha \beta}+B(k r) r_{\alpha} r_{\beta} / r^{2}\right],
$$




$$
\begin{gathered}
A(x)=\left[x^{-1}+i x^{-2}-x^{-3}\right] \exp (i x), \\
B(x)=\left[-x^{-1}-3 i x^{-2}+3 x^{-3}\right] \exp (i x),
\end{gathered}
$$

where $\hat{G} \mathbf{d}=G_{\alpha \beta} d_{\beta}$, the Greek indices stand for the Cartesian components of vectors and summation over repeated indices is implied. The operator $\hat{G}$ is completely symmetrical: $\hat{G}(\mathbf{r})=\hat{G}(-\mathbf{r}), G_{\alpha \beta}(\mathbf{r})=G_{\beta \alpha}(\mathbf{r})$. While the monomer size is assumed to be small compared to the wavelength, the overall cluster size is, in general, arbitrary. That is why the near-, intermediate- and far-zone terms are included in formulas (6),(7).

The CDE is a system of $3 N$ linear equations that can be solved to find the dipole moments $\mathbf{d}_{i}$. The scattering amplitude is expressed through the dipole moments as

$$
\mathbf{f}\left(\mathbf{k}^{\prime}\right)=k^{2} \sum_{i=1}^{N}\left[\mathbf{d}_{i}-\left(\mathbf{d}_{i} \cdot \mathbf{k}^{\prime}\right) \mathbf{k}^{\prime} / k^{2}\right] \exp \left(-i \mathbf{k}^{\prime} \cdot \mathbf{r}_{i}\right)
$$

where $\mathbf{k}^{\prime}$ is the scattered wave vector (which gives the direction of scattering) and $\left|\mathbf{k}^{\prime}\right|=|\mathbf{k}|$. The cross sections of extinction, scattering and absorption can be found from the optical theorem:

$$
\begin{gathered}
\sigma_{e}=\frac{4 \pi}{k} \frac{\operatorname{Im}\left[\mathbf{f}(\mathbf{k}) \cdot \mathbf{E}_{0}^{\star}\right]}{\left|\mathbf{E}_{0}\right|^{2}}=\frac{4 \pi k}{\left|\mathbf{E}_{0}\right|^{2}} \operatorname{Im} \sum_{i=1}^{N} \mathbf{d}_{i} \cdot \mathbf{E}_{i n c}^{*}\left(\mathbf{r}_{i}\right), \\
\frac{d \sigma_{s}}{d \Omega}=|\mathbf{f}(\mathbf{k})|^{2} ; \sigma_{s}=\int|\mathbf{f}(\mathbf{k})|^{2} d \Omega, \\
\sigma_{a}=\sigma_{e}-\sigma_{s} .
\end{gathered}
$$

It can be shown ${ }^{58}$ by direct integration according to (10) over the spatial angles $\Omega$, and with the use of equations (4)-(8), that the integral scattering and absorption cross sections can be expressed through the dipole moments as

$$
\begin{gathered}
\sigma_{s}=\frac{4 \pi k}{\left|\mathbf{E}_{0}\right|^{2}} \sum_{i=1}^{N}\left\{\operatorname{Im}\left[\mathbf{d}_{i} \cdot \mathbf{E}_{i n c}^{*}\left(\mathbf{r}_{i}\right)\right]-y_{a}\left|\mathbf{d}_{i}\right|^{2}\right\} \\
\sigma_{a}=\frac{4 \pi k}{\left|\mathbf{E}_{0}\right|^{2}} y_{a} \sum_{i=1}^{N}\left|\mathbf{d}_{i}\right|^{2}
\end{gathered}
$$




$$
y_{a}=-\operatorname{Im}\left(\frac{1}{\alpha}\right)-\frac{2 k^{3}}{3} \geq 0
$$

Note that the constant $y_{a}$ is non-negatively defined ${ }^{59}$ for any physically reasonable $\alpha$. The ratio $3 y_{a} / 2 k^{3}$ characterizes the relative strength of absorption by a single isolated monomer. However, it was shown ${ }^{59}$ that the resonance absorption of a cluster can be large even when $3 y_{a} / 2 k^{3} \ll 1$.

\subsubsection{Polarizability of a Monomer}

In order to solve the CDE (4), one needs to specify not only the location of monomers, but also the polarizability $\alpha$, which plays the role of the coupling constant. In this Subsection we discuss the methods for defining $\alpha$.

The CDE was originally proposed by Purcell and Pennypacker ${ }^{60}$ for numerical analysis of scattering and absorption of light by nonspherical dielectric particles. In the formulation of Purcell and Pennypacker, a dielectric particle is represented by an array of point dipoles placed on a cubic lattice and restricted by the surface of the particle. The polarizability of an elementary dipole, $\alpha$, is defined by the Clausius-Mossotti relation. Equivalently, it is equal to the polarizability of a little sphere of such a radius that the total volume of all the spheres is equal to the total volume of the particle under investigation. To satisfy this equality, the following relation between the lattice period, $a$, and the radius of a sphere, $R_{m}$, must hold: $a^{3}=4 \pi R_{m}^{3} / 3$. Note that two spheres of the radius $R_{m}$ placed in the neighboring sites of a lattice with the period $a$ would geometrically intersect because $a / R_{m}=(4 \pi / 3)^{1 / 3} \approx 1.612<2$. The polarizability of an elementary dipole in the Purcell-Pennypacker model is given by the Lorenz-Lorentz formula ${ }^{60}$ with the correction for radiative reaction introduced later by Draine: ${ }^{61}$

$$
\begin{gathered}
\alpha=\frac{\alpha_{L L}}{1-i\left(2 k^{3} / 3\right) \alpha_{L L}}, \\
\alpha_{L L}=R_{m}^{3} \frac{\epsilon-1}{\epsilon+2},
\end{gathered}
$$

where $\epsilon$ is the dielectric constant of the material and $\alpha_{L L}$ is the Lorenz-Lorentz polarizability without the radiation correction. Note that the polarizability written in the form (15) provides the positive value of $y_{a}$ (14). More sophisticated formulas for $\alpha$, containing several first terms in the expansion of the polarizability with respect to the parameter $k R_{m}$, can be found in papers by Lakhtakia, ${ }^{62,63}$ Draine and Goodman ${ }^{64}$ and in the references therein. 
In the case of fractal clusters the situation is different from the described above. Instead of the imaginary spheres, we have real touching spherical monomers that form a cluster. Of course, these monomers cannot intersect geometrically. Let the radius of the real monomers that can be seen experimentally be $R_{\exp }$, and the distance between two neighboring monomers be $a_{\text {exp }}$; for touching spheres, $a_{\text {exp }} / R_{e x p}=2$. However, it was shown both theoretically ${ }^{65,66}$ and experimentally ${ }^{67}$ that the CDE (4) with $a_{\text {exp }} / R_{\text {exp }}=2$, $\alpha$ given by (15) and (16), $a=a_{e x p}$ and $R_{m}=R_{e x p}$ yields incorrect results. The reason for this is that the dipole field generated by one monomer inside the other one is not homogeneous; it is much stronger near the point where the monomers touch than in the centers of the neighboring monomer. (Strictly speaking, when the external field is not homogeneous inside a dielectric sphere, one cannot restrict consideration to only dipole moments; the coupled multipole approach is discussed below). Effectively, by replacing two touching spheres by two point dipoles located in their centers, we underestimate the strength of their interaction.

To compensate the above fact, a model for a fractal cluster was introduced $^{68}$ in which neighboring spheres were allowed to intersect geometrically. The radius of these spheres, as well as the distance between two neighboring monomers, are chosen to be different from the experimental ones: $R_{m} \neq$ $R_{\text {exp }}, a \neq a_{\text {exp }}$, but it is required that the ratio $a / R_{m}$ is equal to $(4 \pi / 3)^{1 / 3} \approx$ 1.612 , the same as in the Purcell and Pennypacker model. ${ }^{60}$ Note that a close value for the above ratio, $a / R_{m} \approx 1.688$, was obtained from the condition that an infinite linear chain of polarizable spherules has the correct depolarization coefficient. ${ }^{69}$ The second equation for $R_{m}$ and $a$ can be obtained from the optically important condition that the model cluster has the same fractal dimension, radius of gyration and total volume as the experimental one. The two equations can be satisfied simultaneously for nontrivial fractal clusters (with $D<3$ ) and lead to

$$
R_{m}=R_{\exp }(\pi / 6)^{D /[3(3-D)]} ; \quad N=N_{\exp }(6 / \pi)^{D /(3-D)},
$$

where $N_{\text {exp }}$ and $N$ are the number of monomers in the original and in the model cluster, respectively. The invariance of the radius of gyration, $R_{g}$, follows from (1) and (17).

The above model was shown to be in a good agreement with experimental spectra of fractal cluster. ${ }^{68}$ However, for the practical application of this model, one must take into account that the dielectric constant $\epsilon$ used in formulas (15),(16) is usually tabulated for bulk samples. When very small monomers are considered, a correction to $\epsilon$ connected with the finite-size effects can be significant. ${ }^{68}$ 


\subsubsection{Eigenmode Expansion}

The CDE (4) takes an elegant form when written in matrix notations. We introduce a linear complex vector space $C^{3 N}$ and $3 N$-dimensional vectors of the dipole moments and incident fields according to ${ }^{a}|\mathrm{~d}\rangle=\left(\mathbf{d}_{1}, \mathbf{d}_{2}, \ldots, \mathbf{d}_{N}\right)$ and $|\mathrm{E}\rangle=\left(\mathbf{E}_{\text {inc }}\left(\mathbf{r}_{1}\right), \mathbf{E}_{\text {inc }}\left(\mathbf{r}_{2}\right), \ldots, \mathbf{E}_{\text {inc }}\left(\mathbf{r}_{N}\right)\right)$. We also define an orthonormal basis $|i \alpha\rangle$ in $C^{3 N}$, such that the Cartesian components of the dipole moments are expressed in this basis as $d_{i \alpha}=\langle i \alpha \mid \mathrm{d}\rangle$. The linear operator $\mathrm{W}$ acts on the vector of dipole moments $|\mathrm{d}\rangle$ according to the rule: $\langle i \alpha|\mathrm{W}| \mathrm{d}\rangle=\sum_{\beta j} G_{\alpha \beta}\left(\mathbf{r}_{i}-\right.$ $\left.\mathbf{r}_{j}\right) d_{j \beta}$. Then Eq. (4) can be written as:

$$
|\mathrm{d}\rangle=\alpha(|\mathrm{E}\rangle+\mathrm{W}|\mathrm{d}\rangle)
$$

and the expressions for the optical cross sections acquire the form:

$$
\begin{gathered}
\sigma_{e}=\frac{4 \pi k}{\left|\mathbf{E}_{0}\right|^{2}} \operatorname{Im}\langle\mathrm{E} \mid \mathrm{d}\rangle, \\
\sigma_{a}=\frac{4 \pi k y_{a}}{\left|\mathbf{E}_{0}\right|^{2}}\langle\mathrm{~d} \mid \mathrm{d}\rangle .
\end{gathered}
$$

The interaction matrix $\mathrm{W}$ is complex and symmetrical, and, consequently, non-Hermitian. Therefore, its eigenvectors are not orthogonal, and, moreover, may not form a complete basis in $C^{3 N}$. However, it can be shown ${ }^{58}$ that the eigenvectors are linearly independent (and, therefore, form a basis) if $\mathrm{W}$ is not degenerate, or if the nature of its degeneracy is "geometrical" (due to a certain symmetry of the cluster under consideration) rather than "random". In most practical cases, we can define a complete basis of eigenvectors, $|\mathrm{n}\rangle$, corresponding to the eigenvectors $w_{n}$ :

$$
\mathrm{W}|\mathrm{n}\rangle=w_{n}|\mathrm{n}\rangle .
$$

For complex symmetrical matrices, the orthogonality rule $\left(\langle\mathrm{m} \mid \mathrm{n}\rangle=\delta_{m n}\right)$ is replaced by ${ }^{58}$

$$
\langle\overline{\mathrm{m}} \mid \mathrm{n}\rangle=0 \text { if } m \neq n,
$$

where bar denotes complex conjugation of all elements of a vector. Thus, $|\mathrm{n}\rangle$ denotes a column vector, $\langle\mathrm{n}|$ denotes a row vector with the complex conjugated elements, and $\langle\bar{n}|$ denotes a row vector with exactly the same elements as $|n\rangle$.

\footnotetext{
${ }^{a}$ From this point, we will use Roman letters to denote $3 N$-dimensional vectors and $3 N \times$ $3 \mathrm{~N}$-dimensional matrices, to distinguish them from scalars that are typeset in italics and usual 3-dimensional vectors typeset in bold letters.
} 
We adopt the usual normalization of the eigenvectors, $\langle\mathrm{n} \mid \mathrm{n}\rangle=1$, but $\langle\overline{\mathrm{n}} \mid \mathrm{n}\rangle$ is not equal to unity and can be, in general, complex.

The representation of the unity operator in the basis $|\mathrm{n}\rangle$ is

$$
\mathrm{I}=\sum_{n=1}^{3 N} \frac{|\mathrm{n}\rangle\langle\overline{\mathrm{n}}|}{\langle\overline{\mathrm{n}} \mid \mathrm{n}\rangle} .
$$

The eigenvector expansion for the solution to the CDE, $|\mathrm{d}\rangle$, can be easily obtained with the use of the above equality:

$$
|\mathrm{d}\rangle=\sum_{n=1}^{3 N} \frac{|\mathrm{n}\rangle\langle\overline{\mathrm{n}} \mid \mathrm{E}\rangle}{\langle\overline{\mathrm{n}} \mid \mathrm{n}\rangle\left(1 / \alpha-w_{n}\right)} .
$$

Equations (19),(20), (24) give the general form of the dependence of the solutions to the CDE and the optical cross sections on the polarizability $\alpha$. While the direct numerical application of the eigenvector expansion may be not practical (except for the quasistatic limit, see Section 2.1.3 below), it is useful for analysis of different approximations.

The following two exact properties ${ }^{58}$ of the eigenvalues of the CDE can be useful for assessment of accuracy of different numerical methods for diagonalization of $\mathrm{W}$ :

$$
\sum_{n=1}^{3 N} w_{n}=0 ; \quad-2 k^{3} / 3 \leq \operatorname{Im} w_{n} \leq(3 N-1) 2 k^{3} / 3 .
$$

\subsubsection{Quasistatic Approximation}

If not only the monomers, but the whole cluster is small compared to the incident wavelength $\lambda=2 \pi c / \omega$, one can neglect the intermediate- and far-zone

terms in the interaction operator (5)-(7). Therefore, the matrix $\mathrm{W}$ becomes real and Hermitian:

$$
\left\langle i \alpha\left|\mathrm{W}^{(0)}\right| j \beta\right\rangle=\frac{-\delta_{\alpha \beta}\left|\mathbf{r}_{i}-\mathbf{r}_{j}\right|^{2}+3\left(r_{i \alpha}-r_{j \alpha}\right)\left(r_{i \beta}-r_{j \beta}\right)}{\left|\mathbf{r}_{i}-\mathbf{r}_{j}\right|^{5}},
$$

where $\mathrm{W}^{(0)}$ is the interaction matrix in the quasistatic limit. Analogously, the exponential factor $\exp \left(i \mathbf{k} \cdot \mathbf{r}_{i}\right)$ can be set to unity in the expression for the incident wave (3). The quasistatic free term $\left|\mathrm{E}^{(0)}\right\rangle$ is defined by $\left\langle i \alpha \mid \mathrm{E}^{(0)}\right\rangle=$ $E_{0 \alpha}$. 
The quasistatic eigenvectors, which we denote $\left|\mathrm{n}^{(0)}\right\rangle$, become orthogonal and real. Moreover, as can be easily seen from equation (5), the eigenvectors and the corresponding real eigenvalues, $w_{n}^{(0)}$, do not depend on $\omega$.

Now the eigenvector expansion for the solution of the CDE takes the form

$$
|\mathrm{d}\rangle=\sum_{n=1}^{3 N} \frac{\left|\mathrm{n}^{(0)}\right\rangle\left\langle\mathrm{n}^{(0)} \mid \mathrm{E}^{(0)}\right\rangle}{1 / \alpha-w_{n}^{(0)}}
$$

and can be effectively utilized in numerical calculations. Indeed, the only source of the dependence on the optical frequency $\omega$ in (27) is $\alpha=\alpha(\omega)$. The eigenvectors and the eigenvalues depend only on the geometry of a particular cluster. This means that it is sufficient to diagonalize the interaction matrix $\mathrm{W}^{(0)}$ just once, and then the solution for any frequency can be obtained by a simple summation according to (27).

The quasistatic approximation can be formulated either in the strong or in the weak form. The strong form is obtained by formally setting $k=0$ in formulas (5)-(7). In this approximation, extinction and absorption cross sections are equal, as follows from formulas (14), (19),(20) and the exact relation $\operatorname{Im}\left\langle\mathrm{E}^{(0)} \mid \mathrm{d}\right\rangle=-\operatorname{Im}(1 / \alpha)\langle\mathrm{d} \mid \mathrm{d}\rangle$ that can be found ${ }^{39}$ with the use of $(27)$ :

$$
\sigma_{e}=\sigma_{a}=\frac{4 \pi k}{\left|\mathbf{E}_{0}\right|^{2}} \operatorname{Im} \sum_{n=1}^{3 N} \frac{\left|\left\langle\mathrm{E}^{(0)} \mid \mathrm{n}^{(0)}\right\rangle\right|^{2}}{1 / \alpha-w_{n}^{(0)}} .
$$

Consequently, the scattering cross section is zero in the strong form of the approximation. Physically, the strong form is valid when the absorption parameter $3 y_{a} / 2 k^{3}$ is large, which means that absorption is much larger than scattering.

The strong form of the quasistatic approximation is inadequate for description of scattering. The intuitive idea that the scattering cross section is proportional to the square modulus of the total dipole moment of a cluster (which can be calculated in the assumption that $k=0$ ) was shown to be incorrect. ${ }^{58}$ Also, the strong form of the quasistatic approximation breaks dramatically even for the absorption cross section when the parameter $3 y_{a} / 2 k^{3}$ is not large enough. Although it may seem that the above parameter is always large for small monomers (proportional to $\left.\left(\lambda / R_{m}\right)^{3}\right)$, analysis of formulas (14)(16) shows that this might be not the case when $\operatorname{Im} \epsilon /|\epsilon-1|^{2} \ll 1$. The last inequality can hold either for highly transparent materials $(\operatorname{Im} \epsilon \approx 0)$, or for materials with large $|\epsilon-1|$, such as metals.

In the weak form of the quasistatic approximation, one can still use the eigenvectors $\left|\mathrm{n}^{(0)}\right\rangle$, but it is necessary to keep a few first terms of the expansion 
of $w_{n}$ and $|\mathrm{E}\rangle$ in terms of the powers of $k$. The expansion of the eigenvalues can be done with the use of the standard perturbation theory ${ }^{58}$ using the orthonormal set of $\left|n^{(0)}\right\rangle$ as an unperturbed basis. The results (in the first order of the perturbation theory) are summarized below:

$$
w_{n}=w_{n}^{(0)}+i \frac{2 k^{3}}{3}\left(\left|\mathbf{D}_{n}\right|^{2}-1\right),
$$

where $\mathbf{D}_{n}$ is the total dipole moment of the $n$th eigenmode defined by

$$
D_{n \alpha}=\sum_{i=1}^{N}\left\langle i \alpha \mid \mathrm{n}^{(0)}\right\rangle .
$$

The expansion of the free term is trivial:

$$
\langle i \alpha \mid \mathrm{E}\rangle=E_{0 \alpha}\left[1+i\left(\mathbf{k} \cdot \mathbf{r}_{i}\right)\right] .
$$

It is important to keep the second term in expansion (31) in the case of "antisymmetrical states", ${ }^{58}$ when $\left\langle\mathrm{E}^{(0)} \mid \mathrm{n}^{(0)}\right\rangle=0$.

Finally, the expressions for the optical cross sections are

$$
\begin{gathered}
\sigma_{a}=\frac{4 \pi k}{\left|\mathbf{E}_{0}\right|^{2}} \sum_{n=1}^{3 N}\left|\left\langle\mathrm{E} \mid \mathrm{n}^{(0)}\right\rangle\right|^{2} \frac{y_{a}}{\left|1 / \alpha-w_{n}\right|^{2}} \\
\sigma_{s}=\frac{4 \pi k}{\left|\mathbf{E}_{0}\right|^{2}} \sum_{n=1}^{3 N}\left|\left\langle\mathrm{E} \mid \mathrm{n}^{(0)}\right\rangle\right|^{2} \frac{2 k^{3} / 3+\operatorname{Im} w_{n}}{\left|1 / \alpha-w_{n}\right|^{2}}=\frac{8 \pi k^{4}}{3\left|\mathbf{E}_{0}\right|^{2}} \sum_{n=1}^{3 N}\left|\left\langle\mathrm{E} \mid \mathrm{n}^{(0)}\right\rangle\right|^{2} \frac{\left|\mathbf{D}_{n}\right|^{2}}{\left|1 / \alpha-w_{n}\right|^{2}} .
\end{gathered}
$$

As follows from the analysis of the scattering cross section (33), a cluster cannot be replaced by a single particle with an effective total dipole moment $\mathbf{D}^{t o t}=\sum_{i} \mathbf{d}_{i}$, even when the cluster size is much smaller than $\lambda$. Instead, different eigenmodes scatter independently, without mutual interference. The classical relation $\sigma_{s}=8 \pi k^{4}\left|\mathbf{D}^{t o t}\right|^{2} / 3\left|\mathbf{E}_{0}\right|^{2}$ holds if only one eigenmode can be effectively excited, as in the case of a homogeneous distribution of a large number of monomers inside a spherical volume.

\subsubsection{Born Approximation and Mean Field Theories}

The Born expansion for the solution $|\mathrm{d}\rangle$ can be obtained by iterating equation $(18):^{70,71}$ 


$$
|\mathrm{d}\rangle=\alpha \sum_{k=0}^{\infty}(\alpha \mathrm{W})^{k}|\mathrm{E}\rangle .
$$

It can be easily verified that expansion (34) converges to the exact solution (24) if $\left|\alpha w_{n}\right|<1 \forall n .^{71}$

A rough estimate of the boundaries of the eigenvalues of $\mathrm{W}$ can be made using the Gershgorin theorem which states that each eigenvalue of a matrix $\mathrm{A}=\left\{a_{i j}\right\}$ lies in at least one of the Gershgorin disks $\left|z-a_{i i}\right|<\sum_{j \neq i}\left|a_{i j}\right|$ in the complex $z$-plane. It should be noted that the interaction matrix $\mathrm{W}$ has zero diagonal elements and, therefore, its Gershgorin disks are all concentric. An estimate of the Gershgorin boundaries can be done with the use of a fractal density distribution function, replacing the above summation by integration. The convergence of the Born expansion can strongly depend on the fractal dimension and the cluster size. Especially, this is important when the complete interaction operator (5)-(7) is used. On the other hand, in the quasistatic limit the bounds for eigenvalues are typically independent of the cluster size if $D<3$. This is explained by the fact that the static dipole field is integrable with any reasonable fractal density distribution function at large $r$. For example, quasistatic calculations ${ }^{68}$ for 3 -dimensional lattice cluster-cluster aggregates with the lattice period $a$ showed that $\max \left(\left|a^{3} w_{n}\right|\right) \approx 6$.

The first Born approximation can be used when $\left|\alpha w_{n}\right| \ll 1 \forall n$. Then $|\mathrm{d}\rangle=\alpha|\mathrm{E}\rangle$. Using $\langle\mathrm{E} \mid \mathrm{E}\rangle=N\left|\mathbf{E}_{0}\right|^{2}$, we find that $\sigma_{e}=4 \pi k N \operatorname{Im} \alpha$ and $\sigma_{a}=$ $4 \pi k y_{a} N|\alpha|^{2}$, as one would expect for a collection of non-interacting monomers. Although the integral scattering cross section is given by a formula for $N$ independent scatterers, $\sigma_{s}=\left(8 \pi k^{4} / 3\right) N|\alpha|^{2}$, the differential cross section carries information about the geometrical structure of a cluster. Indeed, as follows from (8) and (10), the differential scattering cross section is proportional to the the function $I(\mathbf{q})$, known as the structure factor: ${ }^{13,28}$

$$
\frac{d \sigma_{s}}{d \Omega} \propto I(\mathbf{q})=\left|\sum_{i=1}^{N} \exp \left(i \mathbf{q} \cdot \mathbf{r}_{i}\right)\right|^{2} ; \mathbf{q}=\mathbf{k}-\mathbf{k}^{\prime} .
$$

For clusters that are spherically symmetrical on average, the structure factor can be averaged over spatial rotations:

$$
\overline{I(\mathbf{q})}=N+N(N-1) \phi_{2}(q) ; \quad \phi_{2}(q)=\int_{0}^{\infty} p_{2}(r) \frac{\sin q r}{q r} d r
$$

where the horizontal line denotes ensemble averaging and $p_{2}(r)$ is the probability density to find two different monomers in a cluster separated by the distance 
$r$. As follows from $(2), p_{2}(r)$ in fractal clusters has a scaling behavior in the intermediate asymptote region $R_{0} \ll r \ll R_{g}: p_{2}(r) \propto r^{D-1}$. If $D<2$ and $q \gg 1 / R_{g}$, the integral in (36) converges at the upper limit while $r$ is still much smaller than $R_{g}$ to produce the well-known result $\overline{I(\mathbf{q})}=N^{2}\left(1 / N+c / q^{D}\right)$, where $c$ is a constant depending on $D$. It was also shown that the dispersion of the average scattered intensity, $\left\langle I^{2}\right\rangle-\langle I\rangle^{2}$, can be expressed similarly to (36) with the use of the fourth-order density correlation function. ${ }^{21,36}$

The mean-field approximation proposed for optical scattering by fractal clusters by Berry and Percival ${ }^{27}$ is physically distinct from the Born expansion, but is very similar mathematically to the first Born approximation. In the mean-field theory, interaction between monomers can be arbitrarily strong and infinite order of multiple scattering is allowed. The main assumption of the mean-field approximation is that the ensemble average of the term $\mathrm{W}|\mathrm{d}\rangle$ in equation (18) can be performed as $\overline{\mathrm{W}|\mathrm{d}\rangle}=\overline{\mathrm{W}} \overline{|\mathrm{d}\rangle}$. In other words, $\mathrm{W}$ and $|\mathrm{d}\rangle$ are assumed to be statistically independent.

For an ensemble of clusters that are spherically symmetrical on average, $\overline{\mathrm{W}}=Q$, where $Q$ is a scalar, because there is no selected direction in space. Performing an ensemble averaging of equation (18), we obtain:

$$
\overline{|\mathrm{d}\rangle}=\frac{\alpha|\mathrm{E}\rangle}{1-Q \alpha}
$$

The above expression is similar to the first term in the Born expansion for $|d\rangle(34)$, except for the constant factor $(1-Q \alpha)^{-1}$. The integral optical cross sections are different in the mean-field approximation from those in the first Born approximation, but the dependence of the scattered intensity on $q=$ $\left|\mathbf{k}-\mathbf{k}^{\prime}\right|$ is exactly the same. Comparison of formula (37) with the exact solution (24) reveals that the mean-field approximation is equivalent to the assumption that all the eigenvalues of $\mathrm{W}$ are equal: $w_{n}=Q$, which, in turn, is possible only if $\mathrm{W}$ is a scalar.

When $|Q \alpha|$ is not small, multiple scattering is important in a cluster. Berry and Percival showed by averaging $\mathrm{W}$ with a fractal distribution function ${ }^{27}$ that the character of multiple scattering is distinctly different when $D<2$ and $D>2$. In the first case, multiple scattering is negligibly small if $k R_{m}$ is much smaller than unity for any cluster size. Physical interpretation of this fact is that a cluster with $D<2$ is always optically transparent. When $D>2$, multiple scattering becomes important if $N \sim 1 /\left(k R_{m}\right)^{D /(D-2)}$, or, equivalently, $R_{g} \sim R_{m} /\left(k R_{m}\right)^{D-2}$. 


\subsection{Coupled Multipoles vs Coupled Dipoles}

The fact that higher multipolar modes must be taken into account when the interacting monomers are close to each other was noted by Gerardy and Ausloos, ${ }^{65}$ Sansonetti and Furdyna ${ }^{67}$ and Claro. ${ }^{66,72,73}$ As was mentioned in Section 2.1.1, the point dipole approximation is inadequate for description of touching spheres. The reason for this is that even if the wavelength of the incident radiation is infinite, the spheres themselves induce highly inhomogeneous fields inside each other which, in turn, excite all the higher multipole moments. It is also incorrect to assume that the fields that act on the point dipoles in the coupled dipoles model are the same as in the centers of the respective spherules; the dipole approximation underestimates the interaction strength between two neighboring monomers.

To overcome the limitations of the coupled dipole approximation, an approach for rigorous numerical solution of the electromagnetic problem of an agglomerate of dielectric spheres interacting with an incident wave and each other has been developed. ${ }^{65,66,72-79}$ We will refer to this approach as to the coupled multipole method. The essence of this method is to expand the EM field inside each sphere, and the field scattered by each sphere, in vector spherical harmonics, and to match the boundary condition on all surfaces of discontinuity. Below, we summarize briefly the mathematical formalism of coupled multipoles.

The fields induced inside the $i$ th sphere, $\mathbf{E}_{i}^{(i n t)}(\mathbf{r})$, and scattered by the $i$ th sphere, $\mathbf{E}_{i}^{(\text {scatt })}(\mathbf{r})$, can be always expanded in the series involving the vector spherical harmonics, $\mathbf{M}_{m n}^{(k)}(\mathbf{r})$ and $\mathbf{N}_{m n}^{(k)}(\mathbf{r})$, where $n$ takes the integer values from 1 to infinity, $m$ takes the integer values from $-n$ to $n$, and the superscript $k$ denotes the type of Bessel function which governs the radial dependence of the corresponding harmonic. In accordance with the commonly accepted convention, $k=1$ corresponds to the spherical Bessel function of the first kind $j_{n}, k=2$ - to the spherical Bessel function of the second kind $y_{n}$, and $k=3,4$ - to the spherical Hankel functions of the first and the second kind, $h_{n}^{(1)}$ and $h_{n}^{(2)}$, respectively. A comprehensive review of the vector spherical harmonics and their properties can be found in the book by Bohren and Huffman. ${ }^{80}$ The expansion for the scattered and internal fields has the following form:

$$
\mathbf{E}_{i}^{(s c a t t)}(\mathbf{r})=\sum_{n=1}^{\infty} \sum_{m=-n}^{n}\left[a_{i, m n} \mathbf{N}_{m n}^{(3)}\left(\mathbf{r}-\mathbf{r}_{i}\right)+b_{i, m n} \mathbf{M}_{m n}^{(3)}\left(\mathbf{r}-\mathbf{r}_{i}\right)\right]
$$




$$
\mathbf{E}_{i}^{(i n t)}(\mathbf{r})=\sum_{n=1}^{\infty} \sum_{m=-n}^{n}\left[c_{i, m n} \mathbf{N}_{m n}^{(1)}\left(\mathbf{r}-\mathbf{r}_{i}\right)+d_{i, m n} \mathbf{M}_{m n}^{(1)}\left(\mathbf{r}-\mathbf{r}_{i}\right)\right]
$$

where $a_{i, m n}, b_{i, m n}, c_{i, m n}$ and $d_{i, m n}$ are the unknown coefficients, and the arguments $\mathbf{r}-\mathbf{r}_{i}$ in the right-hand sides of (38) and (39) reflect the fact that the corresponding spherical harmonics must be calculated in the system of coordinates with the origin at $\mathbf{r}_{i}$; at the same time the functions $\mathbf{M}_{m n}^{(k)}(\mathbf{r})$ and $\mathbf{N}_{m n}^{(k)}(\mathbf{r})$ are universal and independent of the origin. Note that the internal field expansion contains only the spherical Bessel functions of the first kind, $j_{n},(k=1)$ that are finite at $r=0$, while the scattered field expansion contains only the spherical Hankel functions of the first kind, $h_{n}^{(1)}(k=3)$ that have the form of outgoing waves. Expressions for the scattered and internal magnetic field can be obtained by taking a curl of (38) and (39) according to $\mathbf{H}=i k^{-1}$ curlE.

The incident wave is usually supposed to be linearly polarized along the $x$-axis and propagating in the $z$ direction. Then the expansion for $\mathbf{E}_{\text {inc }}(\mathbf{r})=$ $\mathbf{E}_{0} \exp (i \mathbf{k} \cdot \mathbf{r})$ takes the form: ${ }^{80}$

$$
\begin{array}{r}
\mathbf{E}_{i n c}(\mathbf{r})=\exp \left(i \mathbf{k} \cdot \mathbf{r}_{i}\right) \sum_{n=1}^{\infty} \frac{-i^{n+1}\left|\mathbf{E}_{0}\right|(2 n+1)}{2 n(n+1)}\left[\mathbf{M}_{1 n}^{(1)}\left(\mathbf{r}-\mathbf{r}_{i}\right)-\right. \\
\left.\mathbf{M}_{-1 n}^{(1)}\left(\mathbf{r}-\mathbf{r}_{i}\right)+\mathbf{N}_{1 n}^{(1)}\left(\mathbf{r}-\mathbf{r}_{i}\right)+\mathbf{N}_{-1 n}^{(1)}\left(\mathbf{r}-\mathbf{r}_{i}\right)\right] .
\end{array}
$$

The expression in the right-hand side of (40) does not actually depend on $\mathbf{r}_{i}$, but provides an expansion in terms of harmonics centered in the point $\mathbf{r}_{i}$. Note that not all possible spherical harmonics are present in expansion (40). It contains only harmonics with $m= \pm 1$, which is a general property of monochromatic waves. For the scattering problem involving one sphere, only the types of harmonics present in (40) can be excited. However, for the problem involving several spheres, this is not the case, because the secondary scattered waves contain the spherical harmonics of all possible kinds. Again, an expansion for the magnetic field can be obtained by taking a curl of equation (40)

The most challenging task in the coupled multipole method is to expand the spherical harmonics centered around the $i$ th monomer in terms the spherical harmonics centered around the $j$ th monomer. The above procedure is necessary for the boundary conditions consideration, because the field in each 
spherical region must be represented only in terms of spherical harmonics centered in the origin of this region. In general, it is possible to write ${ }^{76}$

$$
\begin{gathered}
\mathbf{M}_{m n}^{(3)}\left(\mathbf{r}-\mathbf{r}_{i}\right)=\sum_{n^{\prime}=1}^{\infty} \sum_{m^{\prime}=-n^{\prime}}^{m^{\prime}=n^{\prime}}\left[A_{m n}^{m^{\prime} n^{\prime}}\left(\mathbf{r}_{i}-\mathbf{r}_{j}\right) \mathbf{M}_{m^{\prime} n^{\prime}}^{(1)}\left(\mathbf{r}-\mathbf{r}_{j}\right)+\right. \\
\left.B_{m n}^{m^{\prime} n^{\prime}}\left(\mathbf{r}_{i}-\mathbf{r}_{j}\right) \mathbf{N}_{m^{\prime} n^{\prime}}^{(1)}\left(\mathbf{r}-\mathbf{r}_{j}\right)\right]
\end{gathered}
$$

An analogous expansion can be written for the outgoing harmonic $\mathbf{M}_{m n}^{(3)}\left(\mathbf{r}-\mathbf{r}_{i}\right)$; it has exactly the same form as (41), but coefficients $A_{m n}^{m^{\prime} n^{\prime}}$ and $B_{m n}^{m^{\prime} n^{\prime}}$ change places. Although the above procedure is simple and physically transparent, the coefficients $A_{m n}^{m^{\prime} n^{\prime}}$ and $B_{m n}^{m^{\prime} n^{\prime}}$ are rather complicated functions of the ClebschGordan coefficients and involve integrals of the Legendre polynomials that cannot be calculated analytically in the general case. We will not adduce them here due to their complexity; the readers interested in numerical implementation of the coupled multipole method can find the necessary coefficients in the original papers by Fuller ${ }^{76,77}$ and $\mathrm{Xu} .{ }^{78}$

For each surface of discontinuity, and for each mode defined by the pair of indices $(m n)$, there are four unknown coefficients, $a_{i, m n}, b_{i, m n}, c_{i, m n}$ and $d_{i, m n}$. Correspondingly, there are four boundary condition equations for the tangential components of the electric and magnetic fields. Note that the internal field in each monomer is given only by expansion (39), while the external field is given by a sum of the incident wave expansion (40) and the sum of all secondary scattered waves (39), where the outgoing spherical harmonics are expanded according to (41). Application of the boundary conditions leads to the following system of linear equations ${ }^{76}$ for the scattering coefficients $a_{i, m n}$ and $b_{1, m n}$ :

$a_{i, m n}=a_{i, m n}^{(0)}\left[p_{i, m n}+\sum_{j \neq i, m^{\prime} n^{\prime}} A_{m n}^{m^{\prime} n^{\prime}}\left(\mathbf{r}_{i}-\mathbf{r}_{j}\right) a_{j, m^{\prime} n^{\prime}}+B_{m n}^{m^{\prime} n^{\prime}}\left(\mathbf{r}_{i}-\mathbf{r}_{j}\right) b_{j, m^{\prime} n^{\prime}}\right]$,

$b_{i, m n}=b_{i, m n}^{(0)}\left[q_{i, m n}+\sum_{j \neq i, m^{\prime} n^{\prime}} B_{m n}^{m^{\prime} n^{\prime}}\left(\mathbf{r}_{i}-\mathbf{r}_{j}\right) a_{j, m^{\prime} n^{\prime}}+A_{m n}^{m^{\prime} n^{\prime}}\left(\mathbf{r}_{i}-\mathbf{r}_{j}\right) b_{j, m^{\prime} n^{\prime}}\right]$,

where $p_{i, m n}$ and $q_{i, m n}$ are both proportional to the exponential factor $\exp (i \mathbf{k}$. $\left.\mathbf{r}_{i}\right)$ ). 
The coupled multipole equation $(42),(43)$ is infinite-dimensional, as the order $n$ can be arbitrary large. Physically, it is usually possible to introduce a reasonable cut-off for $n$. It was shown on the example of a rigorous solution for two touching spheres that such a cut-off can be related to nonlocality effects in the dielectric function, such as a finite wavelength of an electron in a medium. This provides that the harmonics with characteristic features smaller than the electron wavelength cannot be effectively excited. If we denote the cut-off for $n$ as $n_{\max }$, the total number of the coupled multipole equations becomes $N n_{\max }\left(4 n_{\max }+5\right)\left(n_{\max }+1\right) / 6 \sim 2 N n_{\max }^{3}$.

The above fact makes practical application of the coupled multipole method for any system with a reasonably large number of monomers, when the fractal geometry is well manifested, an extremely difficult task. Taking into account that the number of operations necessary to solve a system of $L$ linear equations scales as $L^{3}$ for direct methods and as $L^{2}$ for most iterative methods, the dependence of the computer time on the maximum order $n_{\max }$ is given by $n_{\text {max }}^{6}$ in the most favorable case. Apart from this fact, calculation of the coefficients in equations (42),(43) is a separate computational problem and must be carried out for each wavelength.

When comparing the coupled dipoles and the coupled multipoles methods, we must take into account the following factors. The coupled dipoles formalism treats inaccurately only the interactions between those monomers that are closer to each other than approximately two respective diameters. Thus, most pair interactions in a large cluster are described by the CDE satisfactorily. The model cluster with overlapping neighboring spherules introduced in Section 2.1.1 makes a reasonable correction to the dipole interaction while preserving the geometrical structure, size and total volume of a cluster. The coupled dipole model is very simple from both the theoretical and the computational points of view, with the number of equations scaling as $N$. In the coupled multipoles model, the number of equations scales as $N n_{\text {max }}^{3}$, and it is usually not clear a priori, how large the cut-off value, $n_{\max }$ should be. It was shown ${ }^{75}$ that infinite orders of $n$ are required for the case of two touching spheres, unless nonlocality of the dielectric function is taken into account. However, most calculations based on the coupled multipoles method stop at some relatively small orders of $n$, or involve a relatively small number of spheres. Nevertheless, the coupled multipoles method is very important as an independent rigorous verification of the coupled dipole method and yields some effects that cannot be described in the dipole approximation. 


\subsection{Iterative and renormalization methods}

A number of methods based on the real space renormalization group ${ }^{81,82}$ has been proposed for calculating the optical responses of fractal structures ${ }^{83,84}$ and conductivity of percolation two-component composites. ${ }^{85}$

In the most pure form, the renormalization methods can be applied to geometrical clusters that are mathematically self-similar (unlike the random clusters which are self-similar only in the statistical sense). The geometrical clusters are usually built with the use of a certain "generator" which is replicated at each step to form a larger cluster, which, in turn, is used as a generator for the next step, and so on. ${ }^{83}$ The main idea of the method is to assume that, at each stage, the structure used as a unit for the next stage can be replaced by an equivalent sphere with some polarizability which depends on the unit polarizability at the previous stage and on the geometry. This leads to a recursion relation for the polarizability, or any other optical characteristic, of the generated cluster.

The method of renormalization was successfully applied not only to geometrical clusters, but also to disordered (percolation) systems. ${ }^{84}$

\section{$3 \quad$ Numerical methods}

In this Section we discuss only the numerical methods that can be applied within the coupled dipoles approximation. The numerical implementations of the coupled multipoles and other methods are too vast and diverse and cannot be reviewed here due to the limited volume of this chapter. On the other hand, the coupled dipoles method is most practical when one is interested in the influence of the large scale geometrical structure of a cluster on its optical properties, because the dipole approximation becomes accurate for monomers separated by a distance larger than their size.

\subsection{The quasistatic approximation}

\subsubsection{Jacobi Diagonalization}

In the quasistatic approximation, one of the most powerful numerical approaches is diagonalization of the Hermitian matrix $\mathrm{W}^{(0)}$. This can be done by the standard Jacoby method which proved to be very accurate and robust for clusters with the number of monomers up to $N=1,000$. The diagonalization needs to be done only once for each cluster.

In the strong form of the quasistatic approximation, one can be interested in the spectral dependence of the extinction (or, equivalently, the absorption) cross section. The latter can be calculated with the use of (27) for each $\lambda$ (or 
for each value of $\alpha$ ) by simple summation, once the diagonalization of $\mathrm{W}^{(0)}$ is done. Note that for calculation of the cross sections, it is sufficient to store in memory or on disk only the scalar products $\left\langle\mathrm{E}^{(0)} \mid \mathrm{n}^{(0)}\right\rangle$ and the corresponding eigenvalues instead of the complete set of the eigenvectors. However, in this case the cross sections can be found only for a specific polarization of the incident light, defined by $\left\langle i \alpha \mid \mathrm{E}^{(0)}\right\rangle=E_{0 \alpha}$. A more general approach is to define three vectors $\left|\mathrm{E}_{x}^{(0)}\right\rangle,\left|\mathrm{E}_{y}^{(0)}\right\rangle$ and $\left|\mathrm{E}_{z}^{(0)}\right\rangle$, for the $x, y$ or $z$ polarization of the incident light, respectively, and to store the scalar products of these vectors and $\left|\mathrm{n}^{(0)}\right\rangle$ for each $n$. Then the extinction cross section for the cluster under consideration can be easily calculated for any polarization of the incident light (including elliptical polarization) and any value of $\alpha$ by summation according to (27). We emphasize that the set of values $w_{n}^{(0)}$ and $\left\langle\mathrm{E}_{\alpha}^{(0)} \mid \mathrm{n}^{(0)}\right\rangle$, for $\alpha=x, y, z$ and $n=1, \ldots, 3 N$ can be easily stored on disk for consequent calculations.

In the weak form of the quasistatic approximation, we must use formulas (32) and (33) for the optical cross sections. Therefore, in addition to the above values, one needs to store the value of $\left|\mathbf{D}_{n}\right|^{2}$ for each eigenmode. As follows from (30), $\mathbf{D}_{n}$ is also $\alpha$-independent. Further, in most random systems there are no antisymmetrical eigenstates, such that $\left|\left\langle\mathrm{E}^{(0)} \mid \mathrm{n}^{(0)}\right\rangle\right| \ll\left|\mathbf{E}_{0}\right|$. Then it is possible to replace $|\mathrm{E}\rangle$ in $(32),(33)$ by $\left|\mathrm{E}^{(0)}\right\rangle$. If an antisymmetrical eigenstate exists, one must take into account the second term in expansion (31) which is, actually, $\lambda$-dependent. However, this dependence is trivial $(\sim 1 / \lambda)$ and the scalar product $\left\langle\mathrm{E} \mid \mathrm{n}^{(0)}\right\rangle$ can be easily calculated for any $\lambda$ if it is known for a certain value $\lambda_{0}$ (note that the propagation direction of the incident wave must be specified in this case).

If, however, one is interested in the local fields or dipole moments rather than in the integral cross sections (this is important when nonlinear optical responses must be calculated), the complete set of eigenvectors of $\mathrm{W}^{(0)}$ must be stored. For example, the set of eigenvectors for $N=1,000$ would require $36 \mathrm{Mb}$ of storage. If the disk space is limited, the physical value in question can be calculated by the same program, while the eigenvectors are still in the computer memory. The disadvantage of this approach is that after the program is terminated, an important information contained in the eigenvectors is lost and should a need to find another independent physical value arise, the time extensive part of the calculations would have to be repeated.

\subsubsection{Lanczos Algorithm}

When the dimensionality of the system, $L=3 N$, is very large, the complete diagonalization of $\mathrm{W}^{(0)}$ carries excessive information and is not necessary. Indeed, as was discussed in Section 2.1.4, the Gershgorin bounds for the eigen- 
values do not depend in the quasistatic limit on the number of monomers, $N$. This means that the number of resonance eigenvalues that lie inside the homogeneous width $\Gamma=|\operatorname{Im}(1 / \alpha)|$, such that $\left|\operatorname{Re}(1 / \alpha)-w_{n}^{(0)}\right|<\Gamma$ (see equation (27)), grows approximately proportionally to $N$. Therefore, if one is interested only in integral cross sections rather than in the distribution of local fields, it is sufficient to sample only such number of eigenstates that the density of eigenvalues in the interval $\left(w_{\min }^{(0)}, w_{\max }^{(0)}\right)$ becomes larger than $1 / \Gamma$. A method based on this observation was proposed. ${ }^{68}$ For simplicity, we discuss below the strong form of the quasistatic approximation.

As follows from (18),(28), the extinction and absorption cross sections in the strong form of the quasistatic approximation are given by an average of the form $\left\langle\mathrm{E}^{(0)}\left|\left[1 / \alpha-\mathrm{W}^{(0)}\right]^{-1}\right| \mathrm{E}^{(0)}\right\rangle$. (The matrix $\left[1 / \alpha-\mathrm{W}^{(0)}\right]^{-1}$ is given by the expansion $\sum_{n}\left|\mathrm{n}^{(0)}\right\rangle\left\langle\mathrm{n}^{(0)}\right|\left[1 / \alpha-w_{n}^{(0)}\right]^{-1}$.) It was shown ${ }^{86}$ that the above average can be written as a continued fraction that formally terminates after $L$ levels:

$$
\left\langle\mathrm{E}^{(0)}\left|\left[1 / \alpha-\mathrm{W}^{(0)}\right]^{-1}\right| \mathrm{E}^{0)}\right\rangle=\frac{N\left|\mathbf{E}_{0}\right|^{2}}{1 / \alpha-\eta_{0}-\frac{\beta_{1}^{2}}{1 / \alpha-\eta_{1}-\frac{\beta_{2}^{2}}{1 / \alpha-\eta_{2} \ldots}}} .
$$

The $\eta$ 's and $\beta$ 's are determined by the basic Lanczos recursion relation: ${ }^{87}$

$$
\mathrm{W}\left|\mathrm{u}_{i}\right\rangle=\beta_{i}\left|\mathrm{u}_{i-1}\right\rangle+\eta_{i}\left|\mathrm{u}_{i}\right\rangle+\beta_{i+1}\left|\mathrm{u}_{i+1}\right\rangle,
$$

where $\beta_{0}=0,\left|\mathrm{u}_{-1}\right\rangle=\left|\mathrm{u}_{L+1}\right\rangle=0,\left|\mathrm{u}_{0}\right\rangle=\left|\mathrm{E}^{(0)}\right\rangle$ and $\beta$ 's are calculated from the condition that the vectors $\left|\mathrm{u}_{i}\right\rangle$ are orthonormal. For approximate calculations, the fraction is terminated after $n$ steps, where $n \ll L$, and the last fraction in the sequence has the form

$$
\cdots \frac{\beta_{n-1}^{2}}{1 / \alpha-\eta_{n-1}-\beta_{n}^{2} g(\alpha)}
$$

where the terminator $g(\alpha)$ is taken as the Green's function for a constant chain $^{86}$ of vectors $\left|\mathrm{u}_{i}\right\rangle$ :

$$
\begin{gathered}
g(\alpha)=\frac{1 / \alpha-a-\sqrt{(1 / \alpha-a)^{2}-4 b^{2}}}{2 b^{2}} ; \\
a=\left(w_{\max }^{(0)}+w_{\min }^{(0)}\right) / 2 ; \quad a=\left(w_{\max }^{(0)}-w_{\min }^{(0)}\right) / 4
\end{gathered}
$$

and the minimum and maximum eigenvalues, $w_{\min }^{(0)}$ and $w_{\max }^{(0)}$, can be easily determined by diagonalizing the $n \times n$ symmetric tridiagonal matrix made up of the $\eta$ 's on the diagonal and the $\beta$ 's on the first off diagonal. 
The computationally intensive part of this calculation is the matrix vector multiplication, performed at each level. Therefore the total number of operations required is $\sim L^{2} n$ where $n$ is the number of levels in the continuous fraction (44). The number of iterations required for convergence is proportional to $1 / \Gamma$, which seems to be the common property of many iterative methods applied to the CDE.

\subsection{Beyond the quasistatic approximation}

Beyond the quasistatic limit, the diagonalization of the interaction matrix is computationally inefficient because its eigenvalues and eigenvectors depend explicitly of $\lambda$. Therefore, the computationally intensive part must be repeated for any new wavelength. On the other hand, inversion of matrix is a somewhat simpler task than resolving the eigenproblem. Below, we review a direct and an iterative method for inversion of the complex symmetrical matrix that proved to be robust and efficient for the solution of the CDE.

\subsubsection{Method of the Square Root}

An excellent direct method for inversion of a complex symmetrical matrix is the LU expansion, known also as the method of the square root. We summarize it briefly below.

Consider an $L \times L$ symmetrical matrix $\mathrm{A}=\left\{a_{i j}\right\}$ with complex elements ${ }^{b}$. It is always possible to represent this matrix as a product of a lower and upper triangular matrices (hence, the term LU expansion) as $\mathrm{A}=\mathrm{T}^{\prime} \mathrm{T}$, where $t_{i j}=0$ if $j>i$, and prime denotes transposition. An iterative relation for the elements $t_{i j}, j \leq i$ can be easily derived from the equation

$$
a_{i j}=\sum_{k=1}^{\max (i, j)} t_{i k} t_{j k}
$$

Starting with

$$
t_{11}=\sqrt{a_{11}} ; t_{i 1}=a_{i 1} t_{11}^{-1} \text { for } i>1,
$$

and using the recursion

$$
t_{j j}=\sqrt{a_{j j}-\sum_{k=1}^{j-1} t_{j k}^{2}} ; \quad t_{i j}=\left[a_{i j}-\sum_{k=1}^{j-1} t_{i k} t_{j k}\right] t_{j j}^{-1} \text { for } i>j,
$$

\footnotetext{
${ }^{b}$ In our case, $\mathrm{A}=1 / \alpha-\mathrm{W}$ and $L=3 N$.
} 
one can find all the elements $t_{i j}$ in approximately $L^{3} / 6$ floating-point operations. There is also $L$ calculations of a square root involved, but this number is insignificant. Note also that all the elements $t_{j j}$ are not equal to zero if the determinant of $\mathrm{A}$ is not equal to zero. The first step in the iterative process $(50),(51)$ is also always possible because $a_{11}=1 / \alpha$ and, consequently, $t_{11} \neq 0$.

Once the elements $t_{i j}$ are found, the equation $\mathrm{T}^{\prime} \mathrm{T}|\mathrm{x}\rangle=|\mathrm{b}\rangle$ can be easily solved in two steps. First, we denote $|\mathrm{y}\rangle \equiv \mathrm{T}|\mathrm{x}\rangle$ and solve $\mathrm{T}^{\prime}|\mathrm{y}\rangle=|\mathrm{b}\rangle$ for $|\mathrm{y}\rangle$ in $L^{2}$ steps (this can be easily done because the matrix $\mathrm{T}^{\prime}$ is triangular with all the elements above the main diagonal equal to zero). Second, the equation $\mathrm{T}|\mathrm{x}\rangle=|\mathrm{y}\rangle$ is solved for $|\mathrm{x}\rangle$ in exactly the same way.

Because the method of the square root utilizes effectively the symmetry of A, it is approximately 6 times faster than the standard Gaussian method or any other direct method with $\approx L^{3}$ operations. It is also important that the matrices $\mathrm{A}$ and $\mathrm{T}$ can be stored in the same array, which allows to reduce memory equirements.

\subsubsection{Conjugate Gradient Method}

The conjugate gradient method was applied by Draine ${ }^{61}$ in the Purcell-Pennypacker model for interstellar graphite grains and later by Draine and Flatau. ${ }^{88}$ The algorithm of Draine is a special case of a more general conjugate gradient algorithm described by Petravic and Kuo-Petravic. ${ }^{89}$ The implementation of Draine of the conjugate gradient method proved to be very robust for the particular problem of the coupled dipoles equation, and a number of improvements that can increase the computation speed by 2 or 3 times has been reported..$^{90,91}$

The conjugate gradient method is based on minimizing the functional $F(|\mathrm{x}\rangle)=\| \mathrm{A}|\mathrm{x}\rangle-|\mathrm{b}\rangle \|$, where $|\mathrm{x}\rangle$ is the unknown vector and $|\mathrm{b}\rangle$ is the free term. At each step, the components of $|\mathrm{x}\rangle$ are changed along the gradient of $F$ in the complex $L$-dimensional space. Unlike some simpler methods based on the fastest descent in the $L$-dimensional space, the conjugate gradient method always formally converges to the exact solution after $L$ steps; however, it can accurately approximate the solution much earlier. Similar to most other iterative methods, the convergence of the conjugate gradient method depends on the spectral range of the interaction matrix, $w_{\max }-w_{\min }$, and the quality of resonance of an isolated particle, $1 / \Gamma$. The general rule is that the number of iterations must be not smaller $\operatorname{than}^{c}\left(w_{\max }-w_{\min }\right) / \Gamma$. While the value of $\Gamma=|\operatorname{Im}(1 / \alpha)|$ depends only on the material properties, $\left(w_{\max }-w_{\min }\right) / \Gamma$ is

\footnotetext{
${ }^{c}$ The convergence can be much faster in the cases with special symmetry, when many eigenvectors of $\mathrm{A}$ are orthogonal to $|\mathrm{b}\rangle$. If $|\mathrm{b}\rangle$ coincides with one of the eigenvectors, the convergence takes place in just one iteration.
} 
defined by the geometry. Typically, the latter value is larger in more dense structures with higher fractal dimension.

The basic conjugate-gradient algorithm was described in detail by Draine. ${ }^{61}$ To find a solution to the equation $\mathrm{A}|\mathrm{x}\rangle=|\mathrm{b}\rangle$, we start with an initial guess $\left|\mathrm{x}_{0}\right\rangle$ and make the following initial settings:

$$
\begin{array}{r}
|\mathrm{z}\rangle=\mathrm{A}^{\dagger}|\mathrm{b}\rangle \\
\left|\mathrm{p}_{0}\right\rangle=\left|\mathrm{g}_{0}\right\rangle=|\mathrm{z}\rangle-\mathrm{A}^{\dagger} \mathrm{A}\left|\mathrm{x}_{0}\right\rangle \\
\left|\mathrm{w}_{0}\right\rangle=\mathrm{A}\left|\mathrm{x}_{0}\right\rangle, \\
\left|\mathrm{v}_{0}\right\rangle=\mathrm{A}\left|\mathrm{p}_{0}\right\rangle
\end{array}
$$

where $\dagger$ denotes Hermitian conjugation. Then, starting with $i=0$, the consequitive orders of approximation are calculated according to

$$
\begin{array}{r}
\alpha_{i}=\left\langle\mathrm{g}_{i} \mid \mathrm{g}_{i}\right\rangle /\left\langle\mathrm{v}_{i} \mid \mathrm{v}_{i}\right\rangle, \\
\left|\mathrm{x}_{i+1}\right\rangle=\left|\mathrm{x}_{i}\right\rangle+\alpha_{i}\left|\mathrm{p}_{i}\right\rangle, \\
\left|\mathrm{w}_{i+1}\right\rangle=\left|\mathrm{w}_{i}\right\rangle+\alpha_{i}\left|\mathrm{v}_{i}\right\rangle, \\
\left|\mathrm{g}_{i+1}\right\rangle=|\mathrm{z}\rangle-\mathrm{A}^{\dagger}\left|\mathrm{w}_{i+1}\right\rangle, \\
\beta_{i}=\left\langle\mathrm{g}_{i+1} \mid \mathrm{g}_{i+1}\right\rangle /\left\langle\mathrm{g}_{i} \mid \mathrm{g}_{i}\right\rangle, \\
\left|\mathrm{p}_{i+1}\right\rangle=\left|\mathrm{g}_{i+1}\right\rangle+\beta_{i}\left|\mathrm{p}_{i}\right\rangle, \\
\left|\mathrm{v}_{i+1}\right\rangle=\mathrm{A}\left|\mathrm{g}_{i+1}\right\rangle+\beta_{i}\left|\mathrm{v}_{i}\right\rangle .
\end{array}
$$

The choice of the initial guess is important for the convergence. One possibility is to use the first Born approximation as the initial guess. If the calculation is repeated for a sequence of $\lambda$ 's, the result of the previous calculation can be used as the initial guess for the next one. This technique can effectively increase the number of points in $\lambda$ calculated in a given time. To further improve the convergence, a correction to the initial guess can be made to account for a change in $\lambda$. For example, a solution $\left|\mathrm{d}_{1}\right\rangle$ corresponding to $\lambda=\lambda_{1}$ is used as an initial guess for the calculation with $\lambda=\lambda_{2}$. The corrected components of the initial guess can be found according to $\left\langle i \alpha \mid \mathrm{d}_{2}^{\text {initial }}\right\rangle=\left\langle i \alpha \mid \mathrm{d}_{1}\right\rangle \exp \left[i\left(\lambda_{1} / \lambda_{2}-\right.\right.$ 1) $\left.\mathbf{k}_{1} \cdot \mathbf{r}_{i}\right]$.

In some cases the convergence of integral characteristics, such as the optical cross sections, is an insufficient indication of reaching a good approximation to the solution. The discrepancy of the equation must be controlled independently. The functional $F(|\mathrm{~d}\rangle) /\langle\mathrm{E} \mid \mathrm{E}\rangle$ can serve as a convenient measure of the discrepancy and should be compared to unity. We'll see below on the example 
of a calculation which uses the solution for a slightly different $\lambda$ as the initial guess that the convergence of the cross sections can be reached significantly earlier than the discrepancy becomes much smaller than unity.

\section{Linear Optical Properties}

Below we illustrate the theoretical and computational approaches discussed in the previous sections by some numerical examples.

\subsection{Calculations in the Quasistatic Limit}

We start with the coupled dipoles calculations of linear optical characteristics in the quasistatic limit. First, we introduce some useful variables. The spectral variable is defined as $-\operatorname{Re}(1 / \alpha)$. In the vicinity of an isolated resonance the polarizability $\alpha$ can be written as

$$
\alpha=\frac{R_{m}^{3} \omega_{m}}{\left(\omega-\omega_{0}\right)-i \gamma},
$$

where $\omega_{0}$ is the resonance frequency and $\gamma$ is the spectral width of the resonance. The quantity $\Gamma$ introduced above is related to $\gamma$ by $\Gamma=\left(\gamma / \omega_{m}\right) R_{m}^{-3}$. As follows from (63), the spectral variable is expressed as $-\operatorname{Re}(1 / \alpha)=(\omega-$ $\left.\omega_{0}\right) / \omega_{m} R_{m}^{3}$ and is proportional to the detuning from the resonance. The introduced spectral variable provides a convenient way to describe the system in the most general terms, without specifying the material properties.

All the clusters used in our calculations were built on a simple cubic lattice with a unit step. This means that the physical quantities of the dimensionality of length, such as $R_{m}$, are measured in lattice units. In particular, this concerns the polarizability $\alpha$ and the eigenvalues $w_{n}^{(0)}$. In fact, we will use below the dimensionless values $a^{3} / \alpha$ and $a^{3} w_{n}^{(0)}$, where the lattice unit $a$ is set to unity.

We also introduce the average polarizability of a cluster as a whole, $\chi$, according to $\chi=\left\langle\mathrm{E}^{(0)}\left|\left[1 / \alpha-\mathrm{W}^{(0)}\right]^{-1}\right| \mathrm{E}^{(0)}\right\rangle$. The extinction cross section is given by $\sigma_{e}=\left(4 \pi k /\left|\mathbf{E}_{0}\right|^{2}\right) \operatorname{Im} \chi$ according to (18) and (19).

In Fig. 1 we show the results of the quasistatic calculations for the value $\operatorname{Im} \chi$ as a function of the spectral variable $-\operatorname{Re}(1 / \alpha)$. The calculation was performed for several ensembles of 3-dimensional cluster-cluster aggregates with $D \approx 1.78$. Calculations for the first two ensembles that contained random clusters with $N=500$ and $N=1,000$, respectively, were done using the complete Jacoby diagonalization of $\mathrm{W}^{(0)}$ and subsequent use of formula (27). The homogeneous width $\Gamma$ was set to 0.1 in these calculations. As one can see from the figure, the value $\left(w_{\max }^{(0)}-w_{\min }^{(0)}\right) / \Gamma$ was approximately equal to 120 . The 


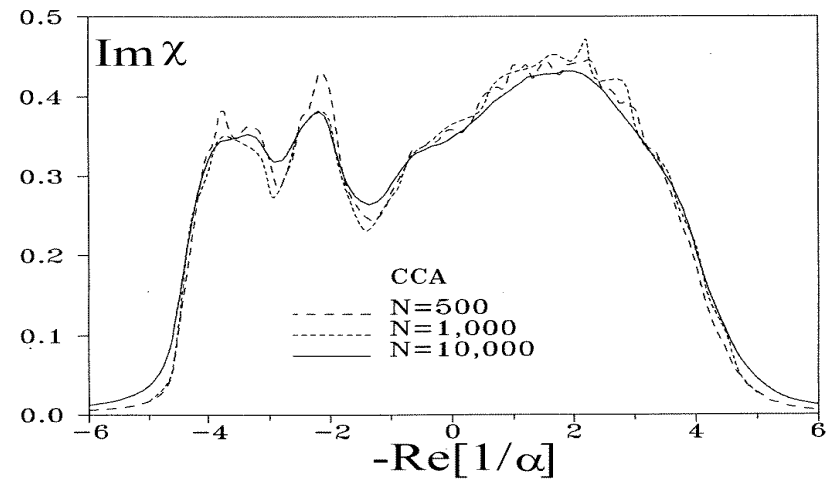

Figure 1: The spectral dependence of the imaginary part of the average cluster polarizability, $\chi$, as a function of the spectral variable $-\operatorname{Re}(1 / \alpha)$ for random cluster-cluster aggregates (CCA's) with different number of monomers, $N$. The curves for $N=500$ and $N=1,000$ are calculated using the Jacobi diagonalization and the curve for $N=10,000$ - using the iterative algorithm based on the Lanczos recursion (Section 3.1.2).

total number of eigenstates in the case of $N=1,000$ was equal to 3,000 and far exceeded the above value.

The third curve in Fig. 1 was calculated using the iterative method based on the Lanczos recursion (Section 3.1.2) for an ensemble of clusters with $N=10,000$. The value of $\Gamma$ was set in this calculation to 0.2 , and the number of iterations was equal to $\approx 100$. We can estimate that $\left(w_{\max }^{(0)}-w_{\min }^{(0)}\right) / \Gamma \approx 60$ in this case, which is not much less than the number of iterations, but a very good agreement between the exact and approximate calculations is achieved. Moreover, the Lanczos calculations are free of the random fluctuations seen in the first two curves, which might appear due to insufficient statistical averaging. We can also conclude that in the quasistatic approximation the spectrum does not depend on the number of particles in a cluster, as long as it is large enough. This can be not so if the complete interaction operator (5)-(7) is used instead of the quasistatic one.

In Fig. 2 we compare the spectra of the imaginary part of the average polarizability, $\operatorname{Im} \chi$, and the density of eigenvalues, $\nu$, calculated as the number of eigenvalues in a unit interval, for cluster-cluster aggregates and for nonfractal random close-packed clusters. The nonfractal clusters were obtained by randomly placing little hard spheres (one after another) in a spherical volume until there was no more available space. This procedure allowed to achieve a fairly high filled volume fraction of $\approx 0.44$ (compare to $\approx 0.52$ for close-packed 


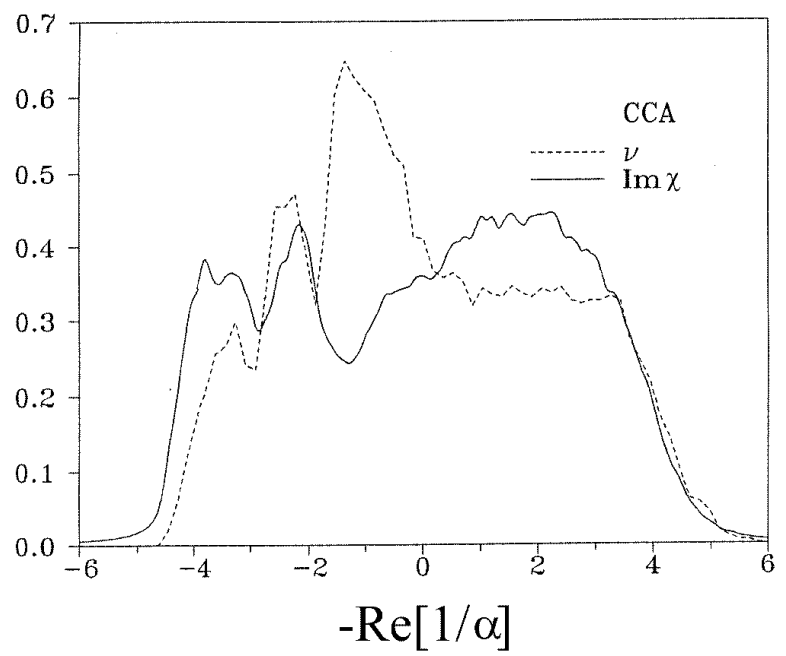

(a)

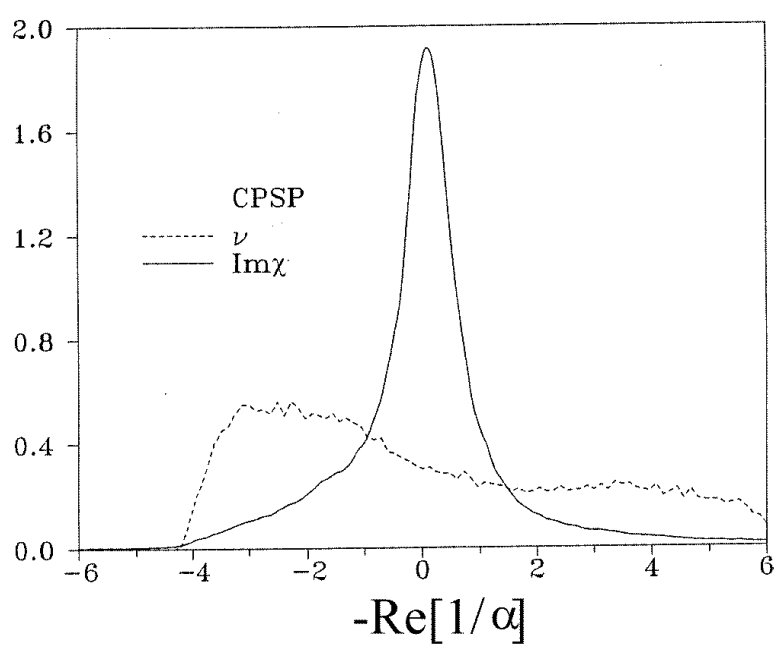

(b)

Figure 2: Spectral dependence of the imaginary part of the average cluster polarizability, $\chi$, as a function of the spectral variable $-\operatorname{Re}(1 / \alpha)$ compared to the density of eigenvalues, $\nu=\nu\left(w^{(0)}=-\operatorname{Re}(1 / \alpha)\right)$. (a): random cluster-cluster aggregate (CCA's); (b): close-packed sphere of particles (CPSP). Calculations are done by the Jacoby diagonalization. 


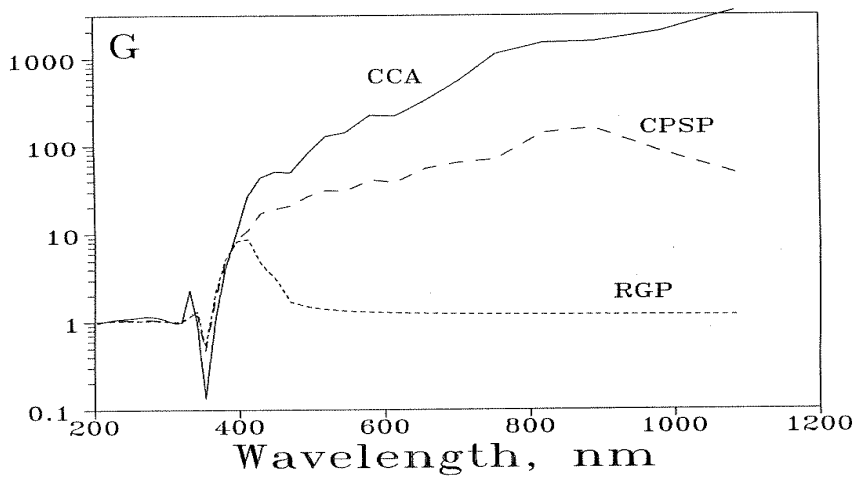

Figure 3: Local field enhancement factor, $G=\overline{\left|\mathbf{E}_{i}\right|^{2}} /\left|\mathbf{E}_{0}\right|^{2}$, as a function of the wavelength for different types of clusters built from silver monomers. CCA - cluster-cluster aggregates $(D \approx 1.78)$; CPSP - close-packed sphere of particles $(D=3)$; RGP - random gas of particles $(D=3)$. Calculations are done by the Jacoby diagonalization.

spheres on a cubic lattice). The main conclusion that can be made from comparison of Figs. 2a and 2b is that in the case of fractal clusters the width of the absorption spectrum is approximately equal to the spectral width of the interaction matrix, $w_{\max }^{(0)}-w_{\min }^{(0)}$, while for the trivial clusters it is much smaller. Physically, this means that selection rules play a more important role in trivial clusters than in fractal ones. While in fractal clusters all eigenmodes can be effectively excited by resonance radiation, in trivial clusters the selection rules suppress excitation of all eigenmodes except for the ones with the resonance frequency close to the resonance frequency of an isolated monomer, $\omega_{0}$.

In Fig. 3 we plot the local field enhancement factor, $G=\overline{\left|\mathbf{E}_{i}\right|^{2}} /\left|\mathbf{E}_{0}\right|^{2}$, as a function of the wavelength for different types of clusters built from silver monomers. Here $\mathbf{E}_{i}$ is the local field at the $i$ th site in a cluster, and the horizontal line denotes statistical averaging. In this calculation, we used experimental optical constants for silver ${ }^{92}$ to calculate the polarizability $\alpha(\lambda)$ with the use of $(15),(16)$ as discussed in Section 2.1.1. Note that the value of $R_{m}$ in (15),(16) was chosen from $a^{3}=(4 \pi / 3) R_{m}^{3}$, and the lattice step, $a$, was set to $8 \mathrm{~nm}$. Three different types of clusters are compared. The first type is the cluster-cluster aggregates (CCA). The second type is the non-fractal close-packed sphere of particles (CPSP) described above. And the third type is also nonfractal random gas of particles (RGP) distributed randomly in a volume that would be occupied by a CCA cluster with the same number of monomers. In all cases, monomers were allowed to approach each other no 
closer than the lattice unit, $a$. Thus, the CPSP's and the RGP's differ only in the occupied volume fraction. In the CPSP model, monomers are randomly placed in a fixed spherical volume until there is some space available, which leads asymptotically to a constant occupied volume fraction, independent of the number of monomers. In the RGP model, the fraction decreases with $N$ and is asymptotically zero.

Fig. 3 demonstrates that the fluctuative nature of fractals results in very strong fluctuations of the local fields. The fluctuations increase towards the infrared. This occurs because both the localization of fractal eigenmodes and their quality factor increase in the long-wavelength part of the spectrum. ${ }^{68}$ This fact is especially important in nonlinear optics where the fluctuations of the local field can play the prevailing role. ${ }^{19,93}$

\subsection{Conjugate Gradient Calculations}

Now, we turn to the calculations beyond the quasistatic limit. Below, we illustrate the conjugate gradient method described in Section 3.2.2. The calculations are performed for a quasi 2-dimensional cluster-cluster aggregate built form 10,000 monomers. The original 3-dimensional CCA was dropped on a plain surface and collapsed so that there is no empty spaces beneath the monomers. This model is relevant to the near-field scanning optical microscopy

of fractals. ${ }^{48}$ For convenience, we consider a reference frame in which the above surface coincides with the $x y$-plane, and the $z$-axis is perpendicular to it. The resultant cluster is not spherically symmetrical, so that one anticipates that its spectrum can depend not only on the polarization of the incident light, but also on its direction of propagation. Note that the last effect is not present in the quasistatic approximation. The cluster is built from silver monomers of the radius $R_{m}=5 \mathrm{~nm}$ and the lattice step is $a=8 \mathrm{~nm}$ (which provides that $\left.a / R_{m} \approx(4 \pi / 3)^{1 / 3}\right)$. Again, the experimental optical constants of silver ${ }^{92}$ were used to calculate $\alpha(\lambda)$.

In Fig. 4 we illustrate the convergence of the conjugate gradient method for different values of $\lambda$. In this particular calculation, the incident light propagated in the $z$-direction and was polarized in the $x$-direction. For $\lambda=$ $420,520,620 \mathrm{~nm}$ and $720 \mathrm{~nm}$, the first Born approximation was used as an initial guess. For $\lambda=800 \mathrm{~nm}$, the initial guess was calculated on an earlier stage using $\lambda=780 \mathrm{~nm}$. For each $\lambda$, the iterations were terminated when the discrepancy of the equation became smaller than $2 \cdot 10^{-3}$. It can be easily seen from Fig. 4 that the convergence of the method becomes slower for larger $\lambda$ 's. This is explained by the fact that the resonance quality factor of silver nanoparticles increases towards the infrared. When the result of a preceding calculation is 


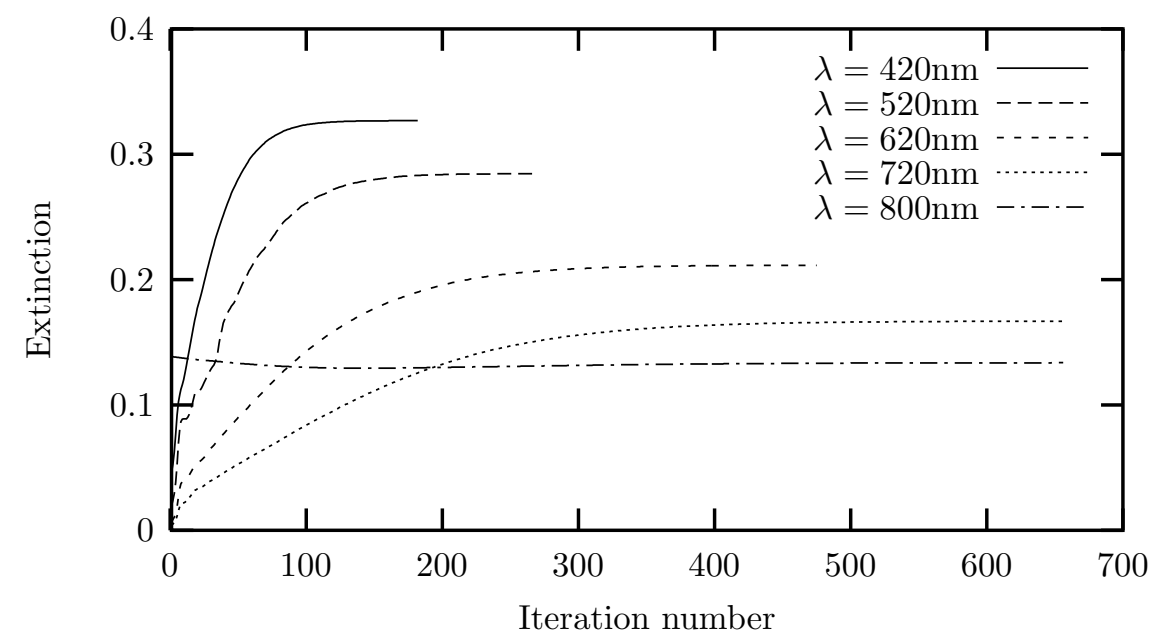

Figure 4: Convergence of the conjugate gradient method for different values of $\lambda$

used as an initial guess, the convergence is, apparently, much faster. However, an independent control of the discrepancy is always required. The calculations showed that while the convergence of the integral cross sections was already achieved, the distribution of local fields was still calculated not accurately enough, and, correspondingly, the discrepancy was large.

In Fig. 5 we plot the spectral dependence of the extinction, absorption and scattering efficiencies, defined as $Q=\sigma / \pi R_{m}^{2} N$ for the cluster described above and for different polarizations and propagation directions of the incident wave. The most characteristic feature of these spectra is the broad long-wavelength wing. An isolated monomer has a resonance peak located near $\lambda=400 \mathrm{~nm}$ with the halfwidth of approximately 50nm. As was discussed in Section 4.1, the external radiation at a certain frequency $\omega \neq \omega_{0}$ can resonancely excite different fractal eigenmodes, while in trivial structures such excitation is prohibited by the selection rules, unless $\omega \approx \omega_{0}$ (here $\omega_{0}$ is the resonance frequency of an isolated monomer). It happens so that in silver clusters many fractal eigenmodes can be excited in the red and near infrared regions. This fact explains the existence of the long-wavelength tails in the spectra in Fig. 5. In the shortwavelength region $(\lambda<300 \mathrm{~nm})$, the nonresonance (bulk) absorption of silver becomes prevailing, and the fractal modes are not excited.

Another characteristic feature is the dependence of the spectra on the po- 


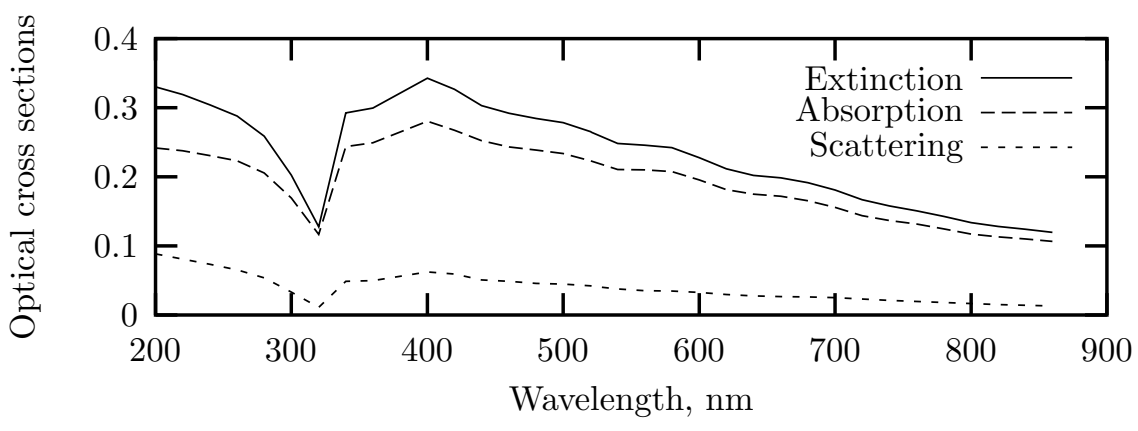

(a)

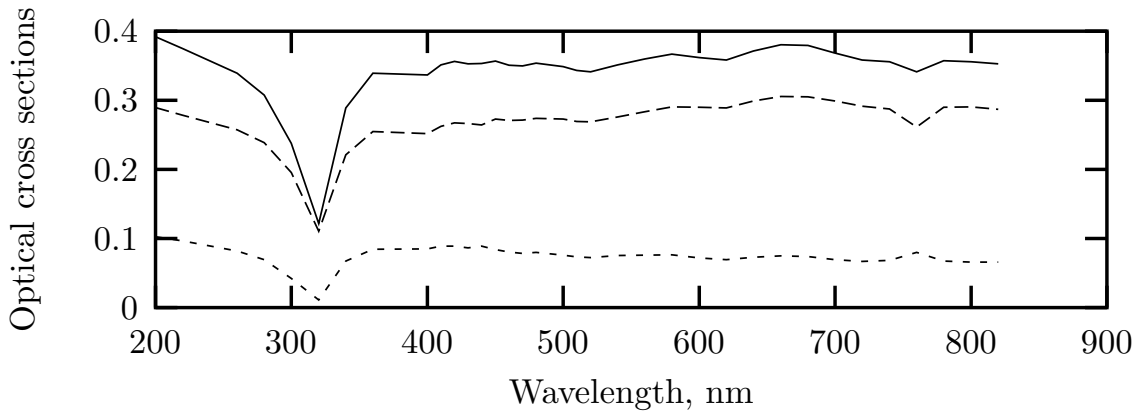

(b)

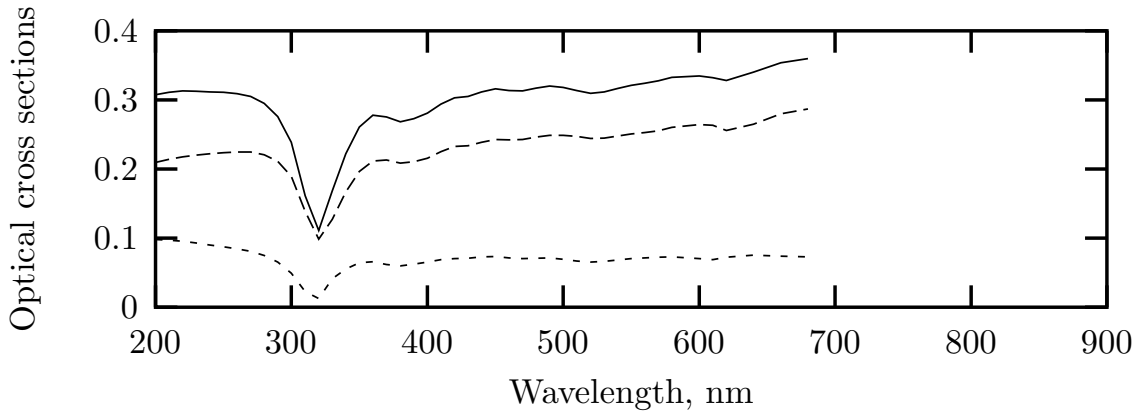

(c)

Figure 5: Extinction, absorption and scattering efficiencies as functions of the wavelength calculated by the conjugate gradient method for different polarizations and propagation directions of the incident light. (a) Propagation - $x$, polarization - $z$; (b) Propagation $-z$, polarization - $x$; (c) Propagation - $y$, polarization - $x$. 


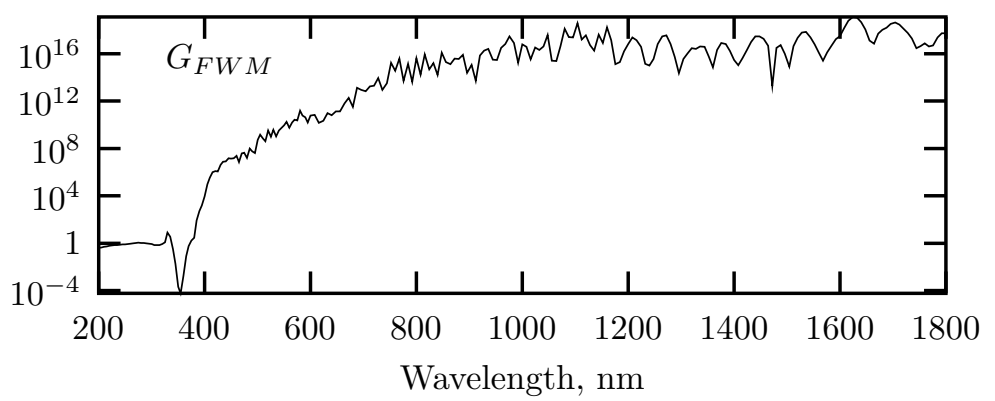

Figure 6: Enhancement factor for the degenerate four wave mixing, $G_{F W M}$ as a function of $\lambda$ for random 3-dimensional cluster cluster aggregates. The calculation was done in the quasistatic limit by Jacoby diagonalization.

larization and propagation direction of the incident light. Note that in Figs. 5b and $5 \mathrm{c}$ the polarization of light is the same (in the surface plane), but the propagation direction is different. The influence of the propagation direction on the optical cross sections is apparent, although the polarization dependence is stronger (compare Figs. 5a and 5b). Note that the dependence on the propagation direction cannot be obtained within the quasistatic approximation.

\section{Nonlinear Optical Properties}

Calculation of local fields is very important in nonlinear optics. Direct computational methods, rather than the iterative ones, are, typically, more appropriate for calculation of the local fields. Many iterative methods, such as the Lanczos-based algorithm described in Section 3.1.2, do not allow calculation of the local fields. Even if they do, as the conjugate gradient method (see Section 3.2.2), it is very difficult to control convergence of the local field at each point in space. Instead, some integral characteristics such as the discrepancy or the convergence of optical cross sections are used to check the accuracy of the obtained solutions. However, even one strong fluctuation in the local field can give a significant input to the generated nonlinear signal.

Below we show the results of quasistatic calculations of the enhancement factor for the degenerate four-wave mixing. This is a nonlinear optical effect of the third order, and the enhancement factor can be defined as ${ }^{93}$ 


$$
G_{F W M}=\left|\frac{\overline{\left|\mathbf{E}_{i}\right|^{2} \mathbf{E}_{i}^{2}}}{\mathbf{E}_{0}^{4}}\right|^{2} .
$$

The calculations were carried out for 3-dimensional cluster-cluster aggregates with $N=1,000$ by the direct Jacoby diagonalization. As above, the clusters were considered to be built from silver monomers of the radius $R_{m}=5 \mathrm{~nm}$ and the experimental optical constants of silver were used.

Because of the high order of the optical nonlinearity involved, the enhancement factor is extremely sensitive to fluctuations in the local field. In the infrared region, where these fluctuations are strong due to high quality of fractal resonances, the average enhancement can reach the enormous value of $10^{16}$ and the local enhancements can reach even much higher values.

\section{Acknowledgments}

This work was supported in part by the National Science Foundation under grants DMR-950050 and DMR-9623663, NATO under the grant CRG950097 and the Environmental Protection Agency under the grant R82265801-0. Also, acknowledgment is made to the donors of the Petroleum Research Fund, administered by the ACS, for partial support of this research.

\section{References}

1. B. B. Mandelbrot, The fractal geometry of nature (Freeman, San Francisco, 1982).

2. Fractals in physics, edited by L. Pietroniero and E. Tosatti (NorthHolland, Amsterdam, 1986).

3. B. Sapoval, Fractals (Aditech, Paris, 1990).

4. R. Botet and R. Jullien, Phase Transitions 24-26, 691 (1990).

5. R. Jullien and R. Botet, Aggregation and fractal aggregates (World Scientific, Singapore, 1987).

6. A. V. Karpov et al., JETP Lett. 48, 571 (1988).

7. A. I. Plekhanov, G. L. Plotnikov, and V. P. Safonov, Opt. Spectrosc. (USSR) 71, 451 (1991).

8. Y. E. Danilova, A. I. Plekhanov, and V. P. Safonov, Physica A 185, 61 (1992).

9. Y. E. Danilova, S. G. Rautian, and V. P. Safonov, Bulletin of the Russian Acad. Sci. - Physics 60, 374 (1996).

10. Y. E. Danilova, V. P. Drachev, S. V. Perminov, and V. P. Safonov, Bulletin of the Russian Acad. Sci. - Physics 60, 342 (1996). 
11. C. W. Bruce, T. F. Stromberg, K. P. Gurton, and J. B. Mozer, Appl. Opt. 30, 1537 (1991).

12. N. Lu and C. M. Sorensen, Phys. Rev. E 50, 3109 (1994).

13. J. Cai, N. Lu, and C. M. Sorensen, J. Colloid Interface Sci. 171, 470 (1995).

14. E. F. Mikhailov and S. S. Vlasenko, Physics-Uspekhi 165, 253 (1995).

15. S. D. Andreev, L. S. Ivlev, E. F. Mikhailov, and A. A. Kiselev, Atmos. Oceanic Opt. 8, 355 (1995).

16. S. D. Andreev and E. F. Mikhailov, Bulletin of the Russian Acad. Sci. 32, 743 (1996).

17. R. Chiarello, V. Panella, and J. Krim, Phys. Rev. Lett. 67, 3408 (1991).

18. J. Krim, I. Heyvaert, C. Van Haesendonck, and Y. Bruynseraede, Phys. Rev. Lett. 70, 57 (1993).

19. V. M. Shalaev, Phys. Rep. 272, 61 (1996).

20. C. Amitrano, A. Coniglio, P. Meakin, and M. Zannetti, Phys. Rev. B 44, 4974 (1991).

21. V. A. Markel, V. M. Shalaev, E. Y. Poliakov, and T. F. George, Phys. Rev. E 55, 7313 (1997).

22. P. Meakin, Phys. Rev. Lett. 51, 1119 (1983).

23. M. Kolb, R. Botet, and R. Jullien, Phys. Rev. Lett. 51, 1123 (1983).

24. T. A. Witten and L. M. Sander, Phys. Rev. Lett. 47, 1400 (1981).

25. D. Stauffer and A. Aharony, Introduction to percolation theory, 2 ed. (Taylor and Francis, Philadelphia, 1991).

26. H. D. Bale and P. W. Schmidt, Phys. Rev. Lett. 53, 596 (1984).

27. M. V. Berry and I. C. Percival, Optica Acta 33, 577 (1986).

28. J. E. Martin and A. J. Hurd, J. Appl. Cryst. 20, 61 (1987).

29. M. Y. Lin et al., J. Colloid Interface Sci. 137, 263 (1990).

30. M. Carpineti, M. Giglio, and V. Degiorgio, Il Nuovo Cimento 16D, 1243 (1994).

31. M. Carpineti, M. Giglio, and V. Degiorgio, Phys. Rev. E 51, 590 (1995).

32. D. Asnaghi, M. Carpiteti, M. Giglio, and A. Vailati, Physica A 213, 148 (1995).

33. F. Sciortino, A. Belloni, and P. Tartaglia, Phys. Rev. E 52, 4068 (1995).

34. N. G. Khlebtsov and A. G. Mel'nikov, Opt. Spectrosc. (USSR) 79, 656 (1995).

35. N. G. Khlebtsov, Colloid J. 58, 100 (1996).

36. V. A. Markel, V. M. Shalaev, E. Y. Poliakov, and T. F. George, J. Opt. Soc. Am. A 14, 60 (1997).

37. V. M. Shalaev and M. I. Stockman, Z. Phys. D 10, 71 (1988).

38. A. V. Butenko, V. M. Shalaev, and M. I. Stockman, Z. Phys. D 10, 81 
(1988).

39. V. A. Markel, L. S. Muratov, M. I. Stockman, and T. F. George, Phys. Rev. B 43, 8183 (1991).

40. V. M. Shalaev, R. Botet, and R. Jullien, Phys. Rev. B 44, 12216 (1991).

41. M. I. Stockman, T. F. George, and V. M. Shalaev, Phys. Rev. B 44, 115 (1991).

42. V. M. Shalaev, M. I. Stockman, and R. Botet, Physica A 185, 181 (1992).

43. V. M. Shalaev, M. Moskovits, A. A. Golubentsev, and S. John, Physica A 191, 352 (1992).

44. M. I. Stockman et al., Phys. Rev. B 46, 2821 (1992).

45. V. M. Shalaev, R. Botet, and A. V. Butenko, Phys. Rev. B 48, 6662 (1993).

46. V. M. Shalaev et al., Physica A 207, 197 (1994).

47. M. I. Stockman, L. N. Pandey, L. S. Muratov, and T. F. George, Phys. Rev. Lett. 72, 2486 (1994).

48. D. P. Tsai et al., Phys. Rev. Lett. 72, 4149 (1994); V. M. Shalaev and M. Moskovits, Phys. Rev. Lett. 75, 2451 (1995).

49. M. I. Stockman, L. N. Pandey, and T. F. George, Phys. Rev. B 51, 185 (1995).

50. M. I. Stockman, L. N. Pandey, and T. F. George, Phys. Rev. B 53, 2183 (1996).

51. V. M. Shalaev, R. Botet, J. Mercer, and E. B. Stechel, Phys. Rev. B 54, 1 (1996).

52. E. Y. Poliakov, V. M. Shalaev, V. A. Markel, and R. Botet, Opt. Lett. 21, 1628 (1996).

53. S. G. Rautian et al., JETP Lett. 47, 243 (1988).

54. A. V. Butenko et al., Z. Phys. D 17, 283 (1990).

55. Y. E. Danilova, V. A. Markel, and V. P. Safonov, Atmos. Oceanic Opt. 6, 821 (1993).

56. S. Alexander and R. Orbach, J. Phys. Lett. (Paris) 43, L625 (1982).

57. S. Alexander, Phys. Rev. B 40, 7953 (1989).

58. V. A. Markel, J. Opt. Soc. Am. B 12, 1783 (1995).

59. V. A. Markel, J. Mod. Opt. 39, 853 (1992).

60. E. M. Purcell and C. R. Pennypacker, Astrophys. J. 186, 705 (1973).

61. B. T. Draine, Astrophys. J. 333, 848 (1988).

62. A. Lakhtakia, Int. J. Mod. Phys. 3, 583 (1992).

63. A. Lakhtakia, Int. J. Infrared Millimeter Waves 13, 869 (1992).

64. B. T. Draine and J. Goodman, Astrophys. J. 405, 685 (1993).

65. J. M. Gerardy and M. Ausloos, Phys. Rev. B 22, 4950 (1980).

66. F. Claro, Phys. Rev. B 25, 7875 (1982). 
67. J. E. Sansonetti and J. K. Furdyna, Phys. Rev. B 22, 2866 (1980).

68. V. A. Markel et al., Phys. Rev. B 53, 2425 (1996).

69. V. A. Markel, J. Mod. Opt. 40, 2281 (1993).

70. P. Chiappetta, J. Phys. A 13, 2101 (1980).

71. S. B. Singham and C. F. Bohren, J. Opt. Soc. Am. A 11, 1867 (1988).

72. F. Claro, Phys. Rev. B 30, 4989 (1984).

73. F. Claro, Solid State Comm. 49, 229 (1984).

74. R. Rojas and F. Claro, Phys. Rev. B 34, 3730 (1986).

75. R. Fuchs and F. Claro, Phys. Rev. B 35, 3722 (1987).

76. K. A. Fuller, J. Opt. Soc. Am. A 11, 3251 (1994).

77. K. A. Fuller, J. Opt. Soc. Am. A 12, 881 (1995).

78. Y.-l. Xu, Appl. Opt. 34, 4573 (1995).

79. T. Lemaire, J. Opt. Soc. Am. A 14, 470 (1997).

80. C. F. Bohren and D. R. Huffman, Absorption and scattering of light by small particles (John Wiley \& Sons, New York, 1983).

81. P. J. Reynolds, W. Klein, and H. E. Stanley, J. Phys. 10, L167 (1977).

82. A. K. Sarychev, Sov. Phys. JETP 45, 524 (1977).

83. F. Claro and R. Fuchs, Phys. Rev. B 44, 4109 (1991).

84. F. Brouers et al., Phys. Rev. B 55, 13234 (1997).

85. J. Bernasconi, Phys. Rev. B 18, 2185 (1978).

86. R. Haydock, in Solid state physics, edited by H. Ehrenreich, F. Seitz, and D. Turnbull (Academic Press, New York, 1980), Vol. 35, Chap. The recursive solution of the Schrodinger equation, pp. 215-294.

87. J. K. Collum and R. A. Willowghby, Lanczos algorithm for large symmetric eigenvalue computations (Birkhauser, Boston, 1985), Vol. 1.

88. B. Draine and P. Flatau, J. Opt. Soc. Am. A 11, 1491 (1994).

89. M. Petravic and J. Kuo-Petravic, J. Comput. Phys. 32, 263 (1979).

90. K. Lumme and J. Rahola, Astrophys. J. 425, 653 (1994).

91. P. J. Flatau, Opt. Lett. 22, 1205 (1997).

92. P. B. Johnson and R. W. Christy, Phys. Rev. B 6, 4370 (1972).

93. V. M. Shalaev, E. Y. Poliakov, and V. A. Markel, Phys. Rev. B 53, 2437 (1996). 